\title{
The role of host phenology for parasite transmission
}

\author{
Hannelore MacDonald ${ }^{1}$ (D) Erol Akçay $^{1} \cdot$ Dustin Brisson $^{1}$
}

Received: 1 April 2020 / Accepted: 20 September 2020 / Published online: 11 November 2020

(c) The Author(s) 2020

\begin{abstract}
Phenology is a fundamental determinant of species distributions, abundances, and interactions. In host-parasite interactions, host phenology can affect parasite fitness due to the temporal constraints it imposes on host contact rates. However, it remains unclear how parasite transmission is shaped by the wide range of phenological patterns observed in nature. We develop a mathematical model of the Lyme disease system to study the consequences of differential tick developmental-stage phenology for the transmission of B. burgdorferi. Incorporating seasonal tick activity can increase B. burgdorferi fitness compared to continuous tick activity but can also prevent transmission completely. $B$. burgdorferi fitness is greatest when the activity period of the infectious nymphal stage slightly precedes the larval activity period. Surprisingly, B. burgdorferi is eradicated if the larval activity period begins long after the end of nymphal activity due to a feedback with mouse population dynamics. These results highlight the importance of phenology, a common driver of species interactions, for the fitness of a parasite.
\end{abstract}

Keywords Disease ecology $\cdot$ Parasite $\cdot$ Seasonality $\cdot$ Phenology $\cdot$ Lyme disease $\cdot$ Epidemiology

\section{Introduction}

Behaviors or traits that vary seasonally, termed phenology in the ecological literature, impact both the type and strength of ecological interactions within populations and communities (Miller-Rushing et al. 2010; Bewick et al. 2016; Paull and Johnson 2014; Barber et al. 2016; Burkett-Cadena et al. 2011). For example, seasonal matching between flowering times and pollinator activity periods is a key driver of short- and long-term population dynamics of both plants and insects (Cleland et al. 2007; Gaku et al. 2004; Inouye 2008; Kudo and Ida 2013; Memmott et al. 2007; Hegland et al. 2009). Differences in the seasonal activities of interacting species over time or geography, caused by changes in climatic and environmental features, can result in population extinctions and in population explosions (Cahill et al. 2013; Johnson et al. 2010; Washburn and Cornell 1981; Powell and Bentz 2009; Jepsen et al. 2009; van Asch and Visser 2007; Jepsen et al. 2008). Although the majority of studies focus on the phenology of plants and their interacting species, the seasonal activity of hosts or disease vectors is also

Hannelore MacDonald

haime@sas.upenn.edu

1 Department of Biology, University of Pennsylvania, Philadelphia, Pennsylvania 19104, USA likely to have large impacts on the population dynamics of infectious microbes.

The impact of phenology on disease transmission dynamics can be prominent in disease systems involving multiple host species or life stages because the seasonal match or mismatch of activities between species or stages will determine the frequency and type of pathogen transmission. For instance, consider the cestode Schistocephalus solidus that infects young three-spined stickleback fish as an intermediate host, multiplies within the fish before the fish is eaten by the definitive bird host (belted kingfisher) (Clarke 1954; Heins et al. 2016). The parasite reproduces sexually within the bird who defecate parasite eggs that infect juvenile fish (Clarke 1954). This disease system occurs in North American lakes that freeze over winter, causing both fish reproduction and bird migration to be temporally restricted within each year. A temporal mismatch in the bird and fish phenologies, such as fish reproduction occurring prior to the return migration of birds, could therefore reduce or eliminate cestode transmission among its hosts. Further, variation in the environmental cues affecting the seasonal activity patterns of the birds and fish either among lakes or across years is likely to impact disease transmission dynamics. These types of seasonal dynamics are expected to impact parasite fitness in many disease systems, yet the quantitative and qualitative 
impact of phenology remains relatively under explored (Barber et al. 2016).

Human diseases caused by zoonotic pathogens, those that complete their natural life cycle in wildlife but can infect humans, are likely impacted by the phenology of their wildlife hosts or vectors. Parasites transmitted by hard bodied ticks (family Ixodidae) represent a practical case study to examine the impact of phenology on disease systems. The public health importance of diseases transmitted by these ticks, such as Lyme disease, has resulted in expansive field datasets that provide baseline expectations for the transmission consequences of tick phenological patterns, making this a good system to study the effects of the general conceptual issue of how vector phenology drives parasite transmission (Randolph 1999b; Randolph et al. 2000; Ogden et al. 2018). Ixodid ticks have three distinct developmental stages. Larvae, the first developmental stage, hatch uninfected but can acquire Borrelia burgdorferi, the etiological agent of Lyme disease, while feeding on an infected host (Fig. 1). Fed larvae molt to nymphs that can then transmit $B$. burgdorferi to small vertebrate hosts (primarily mice, chipmunks, and shrews) during nymphal feeding. Fed nymphs molt to adults that feed on large vertebrates before laying eggs that hatch as larvae. In the Northeastern USA, the nymphal stage is active in early summer while larvae from a different cohort feed in late summer, providing an opportunity for B. burgdorferi transmission from nymphs to larvae through the vertebrate hosts (Wilson and Spielman 1985). This sequential feeding pattern has been alleged to contribute to higher infection prevalence found in the Northeastern USA relative to Southern or Midwestern USA, where the sequential activity patterns are less pronounced (Ogden et al. 2018; Brinkerhoff et al. 2011).

Here we develop a model to study the evolutionary ecology of parasite transmission given different phenological scenarios using the B. burgdorferi-Ixodes tick system as a natural example. The relative simplicity of our model makes mathematical analyses tractable while capturing the fundamental impact of phenology on parasite fitness. This impact unfolds over two timescales: the within-season dynamics of infection and the between-season demography of the vector (Bewick et al. 2016). Previous work (Dunn et al. 2013; Ogden et al. 2004) considered the within-season dynamics of infection but did not account for the between-season population dynamics of the vector species. The latter is an important factor as phenology can alter vector population sizes resulting in an ecological feedback impacting parasite fitness. Our analysis builds on a modeling framework that integrates these effects (Bewick et al. 2016) and demonstrates a general approach for studying both the short- and long-term impacts of vector phenology for

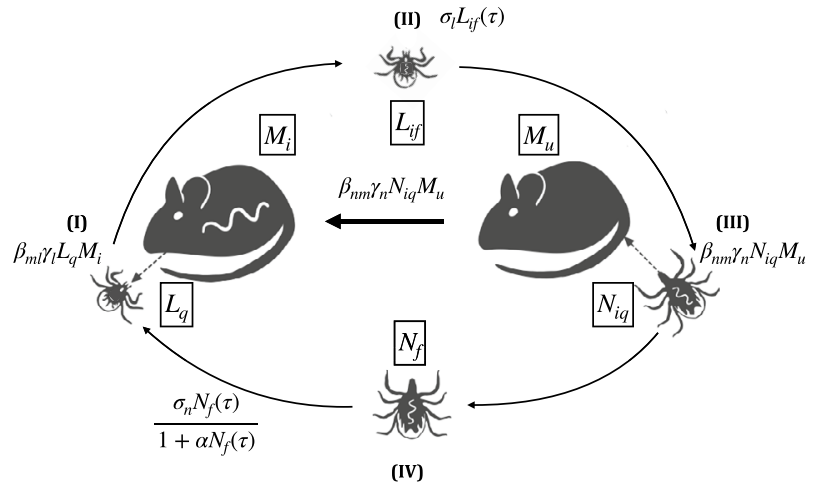

(a)

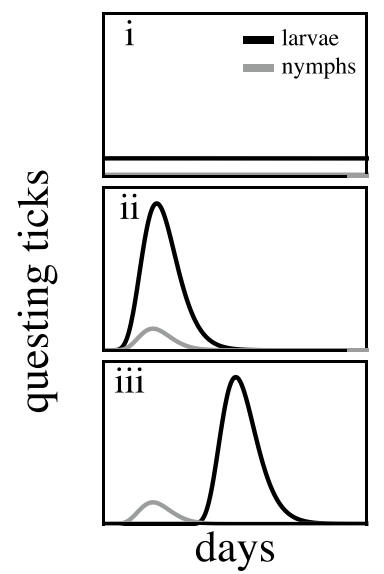

(b)
Fig. 1 How does tick life-stage phenology impact the transmission of $B$. burgdorferi? (a.) Larval ticks hatch uninfected (Piesman et al. 1986; Patrican 1997) and can acquire B. burgdorferi by feeding on an infected small animal (I). Fed infected larvae molt to nymphs and become active the following year (II). Small animals are infected when fed upon by an infected nymph (III). Fed nymphs molt to adults and feed on larger animals prior to laying eggs that hatch the following year $(I V)$. Adult ticks play a minor role in the transmission ecology of B. burgdorferi and are thus not explicitly modeled. Equations describe transitions between tick life stages and infection status from the modeling framework. (b.) The seasonal activity patterns of the tick developmental stages vary from near-continuous activity of all stages throughout the year (i) (Diuk-Wasser et al. 2006; Ogden et al. 2018) to developmental stages with temporally divergent activity seasons of short-duration within the USA (iii) (Diuk-Wasser et al. 2006; Ogden et al. 2018). This latter tick-stage phenology (iii) is thought to result in high transmission of $B$. burgdorferi as large proportions of hosts are infected by nymphs (III) prior to larval activity (I). Black lines show larval activity, and gray lines show nymphal activity. Note that the larvae and nymphs that feed in the same summer are from different cohorts 
parasite fitness. We use the Lyme disease system to describe our approach, although the modeling framework applies to all parasites that require multiple transmission events to complete their life cycle (e.g., West Nile Virus, Leishmania parasites, Yersinia pestis). Our framework can be extended to study how specific vector life history traits, such as differential mortality throughout the year, impact parasite fitness. Additionally, our straightforward framework makes further investigation of the evolutionary pressure imposed by phenology possible.

\section{Model}

We model the transmission of B. burgdorferi between I. scapularis and a main vertebrate reservoir, the white-footed mouse, Peromyscus leucopus (LoGiudice et al. 2003). Our model tracks the within-season dynamics of nymphal and larval population activity and uses these dynamics to compute the between-season changes in overall infection prevalence.

Within-season dynamics describe the duration of nymphal and larval emergence and feeding activity in continuous time from the beginning of each season $(t=0)$ to the end $(t=\tau)$. The life cycle we model is depicted in Figure 1a. Ticks start their life cycle uninfected, but may pick up the infection as larvae by feeding on an infected mouse (Magnarelli et al. 1987). Larvae then overwinter and emerge as nymphs in the next season who can transmit the infection to new mice who are also born uninfected (Hofmeister et al. 1999). The state variables $L_{\bullet}(t), N_{\bullet}(t)$, and $M_{\bullet}(t)$ represent larval, nymphal, and mouse populations, where the subscripts denote the hostseeking status of ticks ( $q$ for questing for a host or $f$ for fed), as well as infection status of ticks and mice ( $i$ for infected, $u$ for uninfected). Thus, $L_{q}$ denotes the questing larvae (who by definition cannot be infected), while $L_{i f}$ denotes fed larvae that are infected. We make the common assumption that mortality and vital rates for both ticks and mice are not impacted by their infection status (Schwanz et al. 2011; Gage et al. 1995). The total mouse population size is $M=M_{i}+M_{u}$. The withinseason dynamics are given by the following system of ordinary differential equations:

$\frac{d L_{q}}{d t}=\hat{L}(T) g_{l}\left(t, l_{l}\right)-L_{q}\left(\gamma_{l} M+\mu_{l}\right)$,

$\frac{d L_{i f}}{d t}=\beta_{m l} \gamma_{l} L_{q} M_{i}$,

$\frac{d L_{u f}}{d t}=\gamma_{l} L_{q}\left(M_{u}+\left(1-\beta_{m l}\right) M_{i}\right)$,

$\frac{d N_{i q}}{d t}=\hat{N}_{i}(T) g_{n}\left(t, l_{n}\right)-N_{i q}\left(\gamma_{n} M+\mu_{n}\right)$, $\frac{d N_{u q}}{d t}=\hat{N}_{u}(T) g_{n}\left(t, l_{n}\right)-N_{u q}\left(\gamma_{n} M+\mu_{n}\right)$,

$\frac{d N_{f}}{d t}=\gamma_{n} M\left(N_{i q}+N_{u q}\right)$,

$\frac{d M_{u}}{d t}=b M(1-M / k)-\mu_{m} M_{u}-\beta_{n m} \gamma_{n} N_{i q} M_{u}$,

$\frac{d M_{i}}{d t}=\beta_{n m} \gamma_{n} N_{i q} M_{u}-\mu_{m} M_{i}$.

Here, $\hat{L}(T)$ represents the total larval population to emerge in year $T$, as determined by the number of nymphs that have successfully fed in the previous year, $T-1$, survived to adulthood, and reproduced (given by equation (18) below). Similarly, $\hat{N}_{i}(T)$ and $\hat{N}_{u}(T)$ represent the total number of questing infected and uninfected nymphs that emerge in year $T$ as determined by the number of infected and uninfected larvae at the end of the previous year and the probability of over-winter survival (see equations (16) and (17)). All other parameters are described in Table 1.

The functions $g_{l}\left(t, l_{l}\right)$ and $g_{l}\left(t, l_{n}\right)$ are probability density functions describing the timing and length of larval and nymphal emergence, respectively. We describe tick emergence using a uniform distribution for analytical tractability:

$g_{\bullet}\left(t, l_{\bullet}\right)=\left\{\begin{array}{cc}0 & t \leq t_{\bullet 0} \\ \frac{1}{l_{0}} & t_{\bullet 0} \leq t \leq t_{\bullet f} \\ 0 & t_{\bullet f} \leq t\end{array}\right.$

where $t_{00}$ is the time emergence begins, $t_{\bullet f}$ is the time emergence stops and $l_{\text {. }}$ is the length of the emergence period $\left(t_{. f}-t_{.0}=l_{\text {e }}\right)$. The uniform distribution establishes a constant emergence probability for ticks over $l_{\text {. and thus spreads }}$ the emergence of the tick cohort evenly across the emergence period from $t_{\text {.f }}<t<t_{\cdot 0}$. While our analysis relies on tick emergence following a uniform distribution, we conducted numerical simulations when tick emergence follows a Gamma distribution and found that the shape of the distribution does not qualitatively change our results (Appendix E.)

Equations (1a-1h) reduce to the following set of equations if we assume that the host population is at equilibrium, $\left(M=k\left(1-\frac{\mu_{m}}{b}\right)\right)$ :

$\frac{d L_{q}}{d t}=\hat{L}(T) g_{l}\left(t, l_{l}\right)-L_{q}\left(\gamma_{l} M+\mu_{l}\right)$,

$\frac{d L_{i f}}{d t}=\beta_{m l} \gamma_{l} L_{q} M_{i}$, 
Table 1 Model parameters and their respective values. Time is measured in days for all parameters

\begin{tabular}{lll}
\hline Parameter & Description & Value \\
\hline$t_{\cdot 0}$ & start of activity period for tick life stage $\bullet$ & varies \\
$t_{\cdot f}$ & end of emergence period for tick life stage $\bullet$ & varies \\
$l$. & length of emergence period for tick life stage $\bullet$ & varies \\
$\hat{L}$ & size of emerging larval population & varies \\
$\hat{N}_{i}$ & size of emerging infected nymphal population & varies \\
$\hat{N}_{u}$ & size of emerging uninfected nymphal population & varies \\
$\gamma_{l}$ & density-dependent contact rate between larvae and mice & 0.004 (Randolph 1999a) \\
$\gamma_{n}$ & density-dependent contact rate between nymphs and mice & 0.008 (Randolph 1999a) \\
$\beta_{n m}$ & transmission probability from nymphs to mice & 0.83 (Davis and Bent 2011) \\
$\beta_{m l}$ & transmission probability from mice to larvae & 0.6 (Davis and Bent 2011) \\
$\mu_{l}$ & larval death rate & 0.015 (Ogden et al. 2005a) \\
$\mu_{n}$ & nymphal death rate & 0.015 (Ogden et al. 2005a) \\
$\mu_{m}$ & mouse death rate & 0.01 (Schug et al. 1991) \\
$b$ & mouse birth rate & 0.1 (Schug et al. 1991) \\
$k$ & mouse carrying capacity & varies (Ostfeld et al. 1996b) \\
$\sigma_{l}$ & larval overwintering survival probability & 0.21 (Davis and Bent 2011) \\
$\sigma_{n}$ & compound fecundity and survival parameter & 10 (Davis and Bent 2011) \\
$\alpha$ & density dependence parameter & 0.0045 \\
$\tau$ & season length & 210 (Ogden et al. 2018) \\
\hline
\end{tabular}

$\frac{d L_{u f}}{d t}=\gamma_{l} L_{q}\left(M-\beta_{m l} M_{i}\right)$,

$\frac{d N_{i q}}{d t}=\hat{N}_{i}(T) g_{n}\left(t, l_{n}\right)-N_{i q}\left(\gamma_{n} M+\mu_{n}\right)$,

$\frac{d N_{u q}}{d t}=\hat{N}_{u}(T) g_{n}\left(t, l_{n}\right)-N_{u q}\left(\gamma_{n} M+\mu_{n}\right)$,

$\frac{d N_{f}}{d t}=\gamma_{n} M\left(N_{i q}+N_{u q}\right)$

$\frac{d M_{i}}{d t}=\beta_{n m} \gamma_{n} N_{i q}\left(M-M_{i}\right)-\mu_{m} M_{i}$

We solve equations (2a-2g) analytically in Appendix B.

\section{Between-season dynamics}

The within-season dynamics described above are coupled to recurrence equations that describe the survival of larvae and nymphs between years. We do not follow the mouse population between years because the impact of overwintering infected mice on $B$. burgdorferi transmission is thought to be negligible (Bunikis et al. 2004; Anderson et al. 1987). The total number of infected and uninfected nymphs $\left(\hat{N}_{i}(T+1)\right.$ and $\left.\hat{N}_{u}(T+1)\right)$ that emerge in a given year are given as a function of the number of infected and uninfected fed larvae at the end of the previous year $\left(L_{i f}(\tau)\right.$ and $\left.L_{u f}(\tau)\right)$ as follows:

$\hat{N}_{i}(T+1)=\sigma_{l} L_{i f}(\tau)$,

$\hat{N}_{u}(T+1)=\sigma_{l} L_{u f}(\tau)$

where $L_{i f}(\tau)$ and $L_{u f}(\tau)$ are the infected and uninfected larval abundances at the end of the previous season (see Appendix B) and $\sigma_{l}$ is the larval overwintering survival probability.

Similarly, the total fed nymphal population at the end of the year $N_{f}(\tau)$ gives rise to the population of larvae, $\hat{L}(T+1)$, that emerges the following year as described by the map:

$\hat{L}(T+1)=\frac{\sigma_{n} N_{f}(\tau)}{1+\alpha N_{f}(\tau)}$

where $N_{f}(\tau)$ is found by integrating (1e) over the season from $(0, \tau)$ as shown in Appendix B. $\sigma_{n}$ is the expected number of eggs produced per fed nymph, after accounting for survival to adulthood and for fecundity. The strength of density dependence on reproduction is determined by $\alpha$.

With these functions, we can write the discrete, betweenseason mapping of the total larval and nymphal abundances from one year to the next: 
$\hat{L}(T+1)=\frac{\sigma_{n} \phi_{n} \hat{N}(T)}{1+\alpha \phi_{n} \hat{N}(T)}$,

$\hat{N}_{i}(T+1)=\sigma_{l} \phi_{l i}\left(\hat{N}_{i}(T)\right) \hat{L}(T)$,

$\hat{N}_{u}(T+1)=\sigma_{l} \phi_{l u}\left(\hat{N}_{i}(T)\right) \hat{L}(T)$

where $\phi_{n}$ denotes the fraction of emerging nymphs that successfully feed as calculated from within-season dynamics $\left(\phi_{n}=\frac{N_{f}(\tau)}{\hat{N}(T)}\right.$; see Appendix A), and $\phi_{l i}\left(\hat{N}_{i}(T)\right)$ and $\phi_{l u}\left(\hat{N}_{i}(T)\right)$ are functions of $\hat{N}_{i}(T)$ that denote the fraction of emerging larvae that become infected or remain uninfected through feeding as calculated from within-season dynamics (e.g., $\phi_{l i}\left(\hat{N}_{i}(T)\right)=\frac{L_{i f}(\tau)}{\hat{L}(T)}$; see Appendix B).

We next calculate the basic reproductive number, $R_{0}$, to quantify the impact of phenology for $B$. burgdorferi fitness. $R_{0}$ represents the average number of new infections caused by a single infected tick in an otherwise naïve population of mice and ticks (McCallum 2001), which gives the threshold for parasite invasibility given the phenology of both tick stages. $R_{0}$ is computed as the number of infected nymphs that emerge in year $T+1$ produced by a single infected nymph that emerged in year $T$ in an otherwise uninfected population. Specifically, we consider a tick population that is at its demographic equilibrium without the infection, solved by setting $\hat{L}^{*}=\hat{L}(T), \hat{N}^{*}=\hat{N}_{u}(T)-1$, and $\hat{N}_{i}(T)=1$ in equations (20)- (21). At this demographic equilibrium, $R_{0}$ of a rare parasite infection is given as follows:

$R_{0}=\frac{\hat{N}_{i}(T+1)}{\hat{N}_{i}(T)}=\sigma_{l} \phi_{l i}\left(\hat{N}_{i}\right) \hat{L}^{*}$

This $R_{0}$ accounts for transmission between cohorts of ticks through intermediate mouse hosts in a given feeding season. When $\hat{N}_{i}(T)=1$, parasites persist in phenological scenarios where $\hat{N}_{i}((T+1)) \geq 1$ (i.e., slope is greater than or equal to unity). Details of the analytical approach are in Appendix C.

\section{Results}

The rate of $B$. burgdorferi transmission from nymphs to mice to larvae is low in systems where either nymphs or larvae are continuously active (Fig. 2). Controlling for total population sizes, when nymphal feeding is evenly spread throughout the year, few nymphs feed at any given time, resulting in limited nymph-to-mouse transmission events. The proportion of infected mice remains constantly low as new infections occur at a similar rate as mouse mortality which replaces older, potentially infected mice with uninfected juveniles. Larval ticks rarely encounter infected mice, thus limiting mouse-to-tick transmission events. By contrast, seasonal nymphal activity concentrates nymphto-mouse transmission events in time, causing a seasonal peak in mouse infection prevalence that decays due to mouse population turnover (Fig. 2). The duration of the nymphal activity period is negatively correlated with the rate at which infected mice accumulate as well as the maximum mouse infection prevalence (e.g., small $l_{n}$ values in Fig. 2). That is, nymphal activity periods of greater duration result in a lower maximum mouse infection prevalence that peaks later in the season (Fig. 2). Larval ticks that feed at or around the peak in mouse infection prevalence are more likely to encounter an infected mouse and to acquire $B$. burgdorferi before molting to nymphs.

The fitness of $B$. burgdorferi, quantified by the basic reproductive number $\left(R_{0}\right)$, is greatest when larval activity is concentrated around the peak in the mouse infection prevalence, thus increasing the probability that each larva will feed on an infected mouse (Fig. 3A. and Fig. 4A).
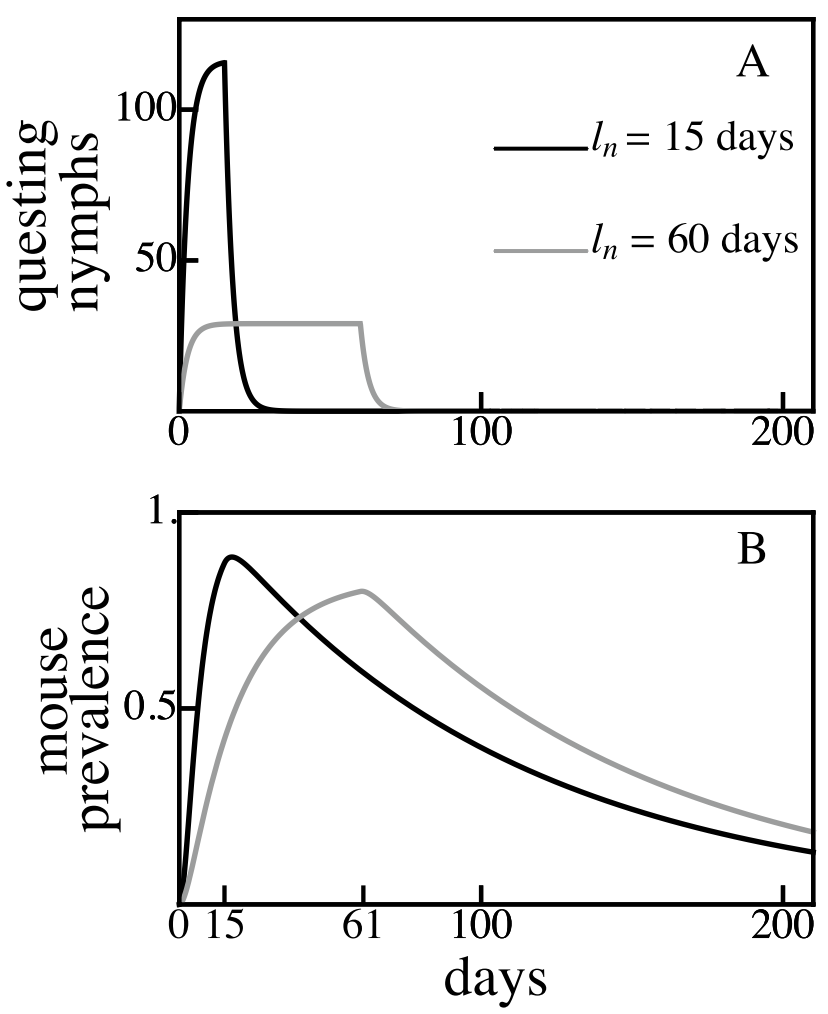

Fig. 2 Concentrated nymphal emergence durations $\left(l_{n}=15\right.$ days, $\left.A\right)$ result in a higher and earlier mouse infection prevalence peak $(B)$ compared to longer nymphal emergence durations $\left(l_{n}=60\right.$ days) for the same total tick population sizes. For example, a nymphal activity duration of 15 days $\left(l_{n}=15\right.$ days, $\left.A\right)$ results in peak mouse infection prevalence $(B)$ occurring on day 15 , while $l_{n}=60$ days results in peak mouse infection prevalence occurring on day 61 . In both models, $25 \%$ of emerging nymphs are infected, $k=50, M=k\left(1-\mu_{m} / b\right)=45$. All other parameter values are shown in Table 1 

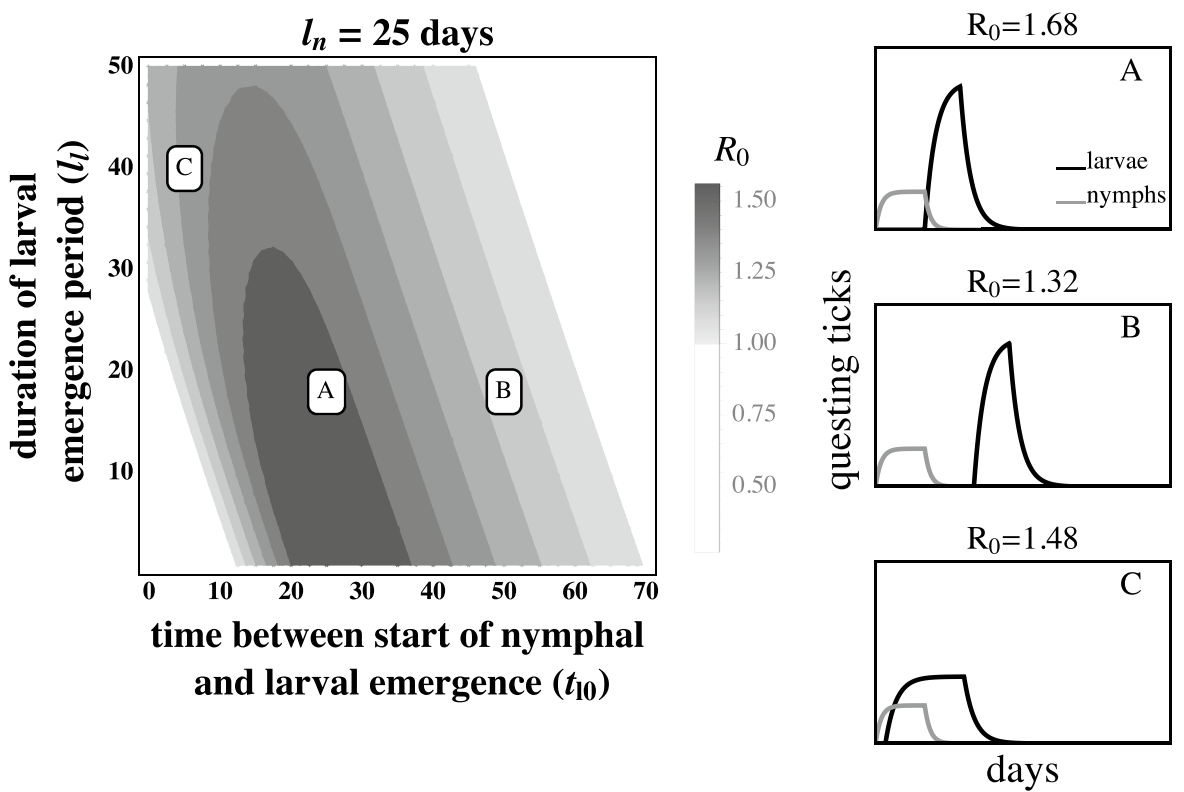

Fig. 3 The basic reproductive number, $R_{0}$, of $B$. burgdorferi is greatest when larval activity is concentrated around peak mouse infection prevalence. The left panel depicts $R_{0}$ as a function of the duration of larval emergence $\left(l_{l}\right)$ and time between nymphal and larval emergence $\left(t_{l 0}\right)$. Panels on the right depict within-season dynamics for representative timing parameter values indicated by their respective letters on the left panel. (A) Concentrated larval emergence (small $\left.l_{l}\right)$ that begins slightly after nymphal emergence $\left(20<t_{l 0}<35\right)$ increases the probability that questing larvae feed on mice recently infected by nymphs $\left(t_{l 0}=25, l_{l}=18\right)$. (B) Transmission decreases as larvae emerge later $\left(t_{l 0}>35\right)$ because the larval cohort feeds after peak mouse infection prevalence $\left(t_{l 0}=50, l_{l}=18\right)$. (C) When larval and nymphal emergence is more synchronous (small $t_{l 0}$ ), transmission to larvae increases as larval emergence duration increases (large $l_{l}$ ) because more larvae feed after infectious nymphs $\left(t_{l 0}=5, l_{l}=40\right)$. B. burgdorferi is not maintained in systems where $R_{0}<1 . R_{0}$ is calculated assuming tick emergence is uniformly distributed $\left(U\left(l_{l}\right)\right.$ where $l_{l}$ is the larval emergence duration, see Appendix C). $\hat{L}=\hat{L}^{*}, \hat{N}_{i}=1, \hat{N}_{u}=\hat{N}^{*}-1$ (see Appendix A.) $l_{n}=25$ days; all other parameter values are shown in Table 1
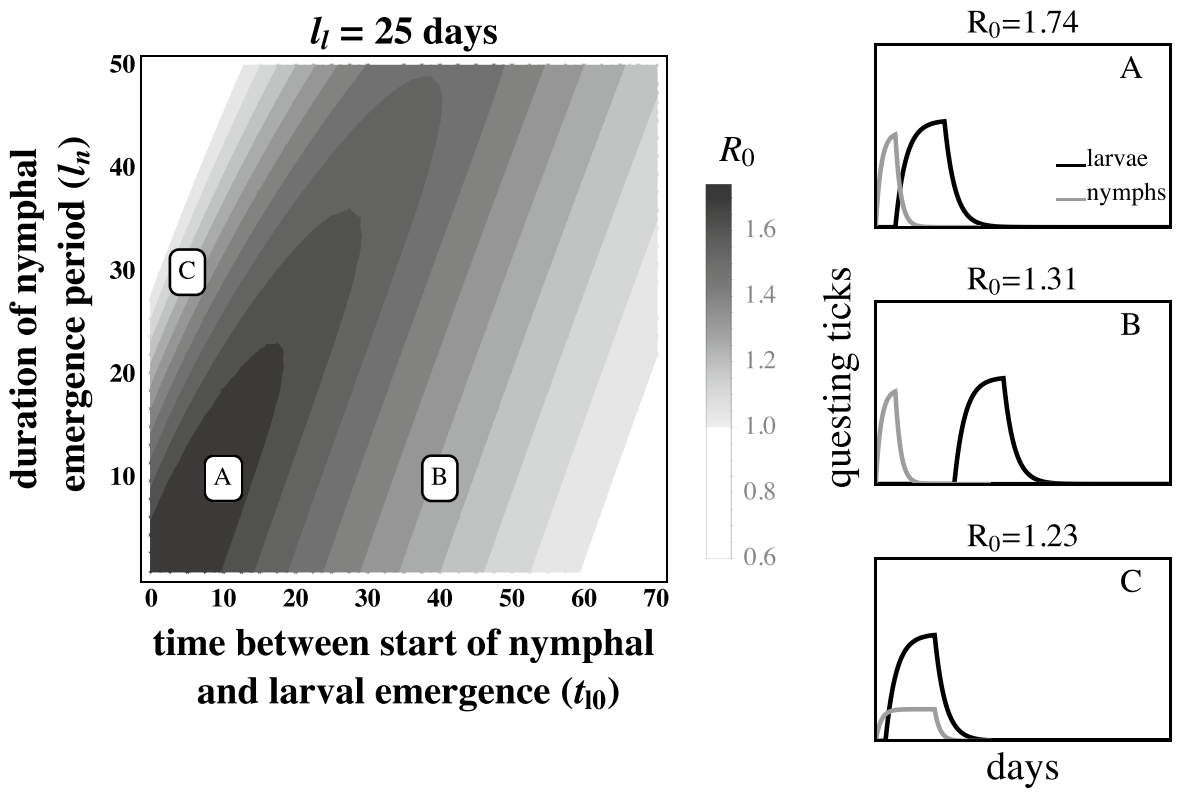

Fig. 4 The basic reproductive number, $R_{0}$, of $B$. burgdorferi is greatest when larval emergence begins shortly after nymphal emergence such that larvae feed during peak mouse infection prevalence. The left panel depicts $R_{0}$ as a function of the time between the start of nymphal and larval emergence $\left(t_{l 0}\right)$ and the duration of the nymphal emergence period $\left(l_{n}\right)$. The letters within the left panel indicate the parameter values used to depict representative within-season dynamics in the right panels. (A) Concentrated nymphal emergence (small $l_{n}$ ) coupled with slight differences in nymphal and larval emergence time $\left(t_{l 0}<10\right)$ increases the probability that questing larvae feed on mice infected by nymphs $\left(t_{l 0}=10, l_{n}=10\right)$. (B) Longer dura- tions between nymphal and larval emergence time $\left(t_{l 0}>10\right)$ result in lower mouse-to-larvae transmission rates as many mice infected by nymphs die and are replaced by mice born uninfected such that larvae are likely to feed on uninfected mice $\left(t_{l 0}=40, l_{n}=10\right)$. (C) Synchronous emergence $\left(t_{l 0}=0\right)$ can also reduce $B$. burgdorferi fitness when nymphal emergence duration is long (large $l_{n}$ ) as many larvae feed before mice become infected by nymphs $\left(t_{l 0}=5, l_{n}=30\right) . R_{0}$ is calculated assuming tick emergence is uniformly distributed $\left(U\left(l_{n}\right)\right.$ where $l_{n}$ is nymphal emergence length, see Appendix C). $l_{l}=25, \hat{L}=\hat{L}^{*}, \hat{N}_{i}=1, \hat{N}_{u}=\hat{N}^{*}-1$ (see Appendix A). All other parameter values are shown in Table 1 

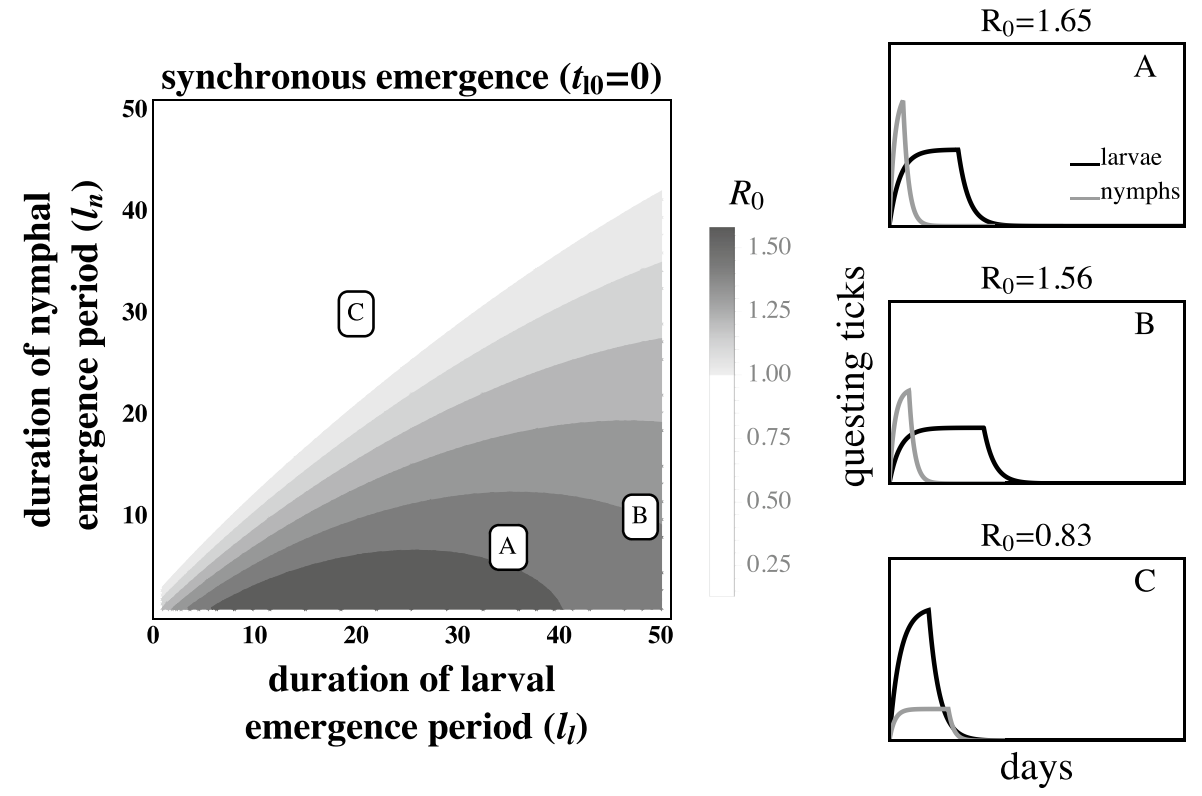

Fig. 5 The larval emergence duration that maximizes $R_{0}$ for $B$. burgdorferi is conditioned on nymphal emergence duration. $R_{0}$ is high if larval emergence duration is slightly longer than nymphal emergence duration $\left(l_{l}>l_{n}\right.$ in $(A)$ and $\left.(B)\right)$, thus allowing larvae to feed on mice that were previously fed upon by nymphs. However, $R_{0}$ decreases when larval emergence duration is much longer than nymphal emergence duration $\left(R_{0}\right.$ of $(B)<R_{0}$ of $\left.(A)\right)$ as late emerging larvae can feed on uninfected mice born after the nymphal activity period. Transmission from mice to larvae is low when the larval emergence duration is less than the nymphal emergence duration $\left(l_{l}<l_{n}\right.$ in $\left.(C)\right)$ because many larvae feed before infectious nymphs. B. burgdorferi is not maintained in systems where $R_{0}<1 . R_{0}$ is calculated assuming tick emergence is uniformly distributed $\left(U\left(l_{l}\right)\right.$ where $l_{l}$ is the larval emergence length. See Appendix $\mathrm{C}$ for details). $\hat{L}=\hat{L}^{*}, \hat{N}^{*}=\hat{N}_{u}-1$ (see Appendix A). $t_{l 0}=0,(A) l_{l}=35, l_{n}=7$ (B) $l_{l}=48, l_{n}=10(C)$ $l_{l}=20, l_{n}=30 . t_{l 0}=0$ days; all other parameter values are shown in Table 1
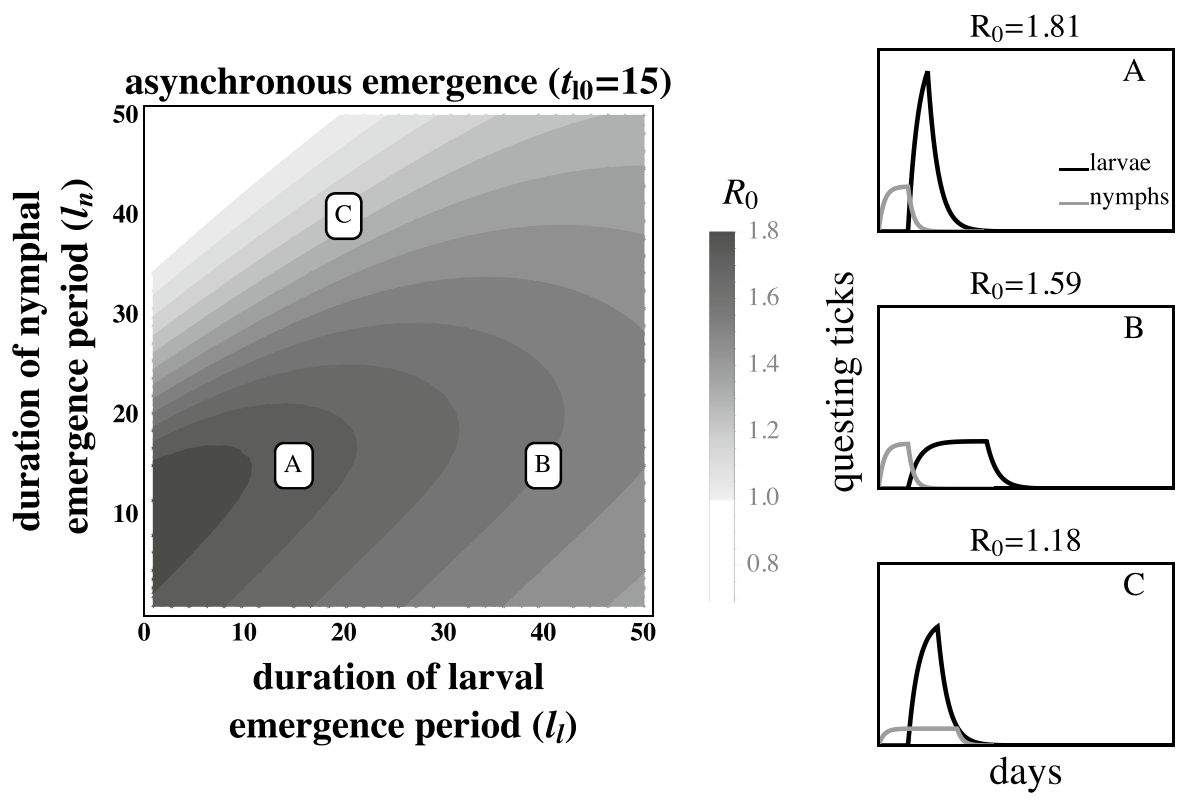

Fig. 6 Highly concentrated larval emergence increases $R_{0}$ when larvae emerge slightly after nymphs. $(A)$ Concentrated nymphal emergence drives high mouse infection prevalence and results in high transmission to larvae when larval emergence is tightly concentrated $\left(l_{l}=15, l_{n}=15\right)$. (B) Transmission from mice to larvae decreases as larval emergence duration increases because larvae are more likely to feed on uninfected mice born after nymphal activity $\left(l_{l}=40, l_{n}=15\right)$.
(C) Transmission from mice to larvae also decreases if larval emergence duration is highly concentrated and nymphal emergence duration is broad because many larvae feed before nymphs infect mice $\left(l_{l}=20, l_{n}=40\right) . R_{0}$ is calculated assuming tick emergence is $U\left(l_{l}\right)$ where $l_{l}$ is the larval emergence length (see Appendix C.) $\hat{L}=\hat{L}^{*}, \hat{N}_{i}=1, \hat{N}_{u}=\hat{N}^{*}-1$ (see Appendix A.) $t_{l 0}=15$ days; all other parameter values are shown in Table 1 
Larvae that are active long after the end of the nymphal activity period are likely to feed on an uninfected mouse due to decays in mouse infection prevalence caused by mouse population turnover (Fig. 3B and Fig. 4B). Similarly, larval activity periods that begin prior to nymphal activity periods result in the majority of larvae feeding on uninfected mice that have not acquired an infection from a feeding nymph.

The effect of larval emergence duration depends on whether or not larval emergence coincides with nymphal emergence: concentrated larval emergence decreases $R_{0}$ when larval and nymphal emergence periods are synchronous (Fig. 5) because most larvae feed before nymphs have a chance to infect the mouse population. Conversely, concentrated larval emergence tends to increase $R_{0}$ when larvae emerge later than nymphs (Fig. 6). This occurs because nymphal emergence that slightly precedes larval emergence results in high mouse infection prevalence when larvae begin emerging (Fig. 6A), and concentrated emergence results in most larvae feeding when the prevalence of infection is still high. In both cases, $R_{0}$ decreases with very broad larval emergence due to mouse turnover (Fig. 3C, Fig. 4C, Fig. 6B, Fig. 5B).

\section{Discussion}

Phenology is a fundamental component of all ecological interactions. Interactions between organisms such as competition, predation, and parasitism are predicated on temporal overlap of interacting species or life stages. Similarly, host or vector phenology impacts parasite fitness by temporally structuring transmission events between interacting hosts or life stages. Host or vector phenological patterns can even determine whether a pathogen is highly abundant or is unable to persist (Fig. 3). The ubiquity of seasonal activity among hosts and vectors, as well as the geographic variation in seasonal activity patterns, underscores the importance of phenology for the distribution and abundance of many pathogenic microbes including malaria, rabies, tapeworm, and Lyme disease (Hoshen and Morse 2004; Gremillion-Smith and Woolf 1988; Anderson 1974; Ogden et al. 2018). Here we derive the basic reproductive number, $R_{0}$, for a transmission dynamics model that explicitly considers the impact of phenology on both parasite transmission and vector demography to assess the impact of vector phenology on parasite transmission and fitness using the Lyme disease system as an example. Our results are benchmarked by field data that show a link between the regional differences in tick phenology and differences in B. burgdorferi distribution and abundance (Ogden et al. 2018). Investigation of parameter space in this model revealed the novel insight that large temporal differences between the activity periods of tick life stages decrease B. burgdorferi fitness.

Our model accounts for an important ecological feedback between vector demography and parasite fitness by incorporating the impact of phenology on demography. This is achieved by capturing both within-season infection dynamics and between-season vector demography in our mathematical analysis. Tick population sizes increase with earlier and more concentrated emergence because most ticks have sufficient time to successfully find a host before the season ends. By contrast, late or less concentrated emergence results in fewer ticks feeding before the season ends as the time available for later-emerging ticks to find a host is effectively shortened. The impact of this demographic feedback is limited at high mouse density but increases rapidly at low mouse density (see Appendix D). Extensions to this framework where vector mortality or contact rates with hosts vary throughout the year are likely to exacerbate the impact of phenology on demography. These results suggest that disregarding between-season demographic dynamics could underestimate parasite fitness $\left(R_{0}\right)$ when ticks emerge early and overestimate $R_{0}$ when ticks emerge later. The importance of this ecological feedback is reflected in the finding from a next-generation model focusing on within-season (but not between-season) impacts of tick phenology on B. burgdorferi fitness which indicated that vector demography is one of the most important model parameters affecting $R_{0}$ (Dunn et al. 2013).

Parasite fitness is maximized when the activity periods of vector life stages are of short duration (Fig. 2). Continuous nymphal activity temporally distributes the finite number of nymph-to-mouse transmission events such that mice become infected at a low rate throughout the season. Mouse infection prevalence remains continually low because mice that die, including infected mice, are replaced by uninfected juveniles at rates similar to the rate at which new infections are introduced. Mouse-to-larvae transmission events are similarly rare as most larvae feed on the relatively abundant uninfected mice. By contrast, seasonal nymphal activity concentrates nymph-to-mouse transmission events leading to many new mouse infections over a short period of time. Mouse infection prevalence increases rapidly during the nymphal activity period, as new infections occur at a much greater rate than mouse mortality, and subsequently decline when new infections stop at the end of the nymphal activity period (Fig. 2). Transmission from mice to larvae is very high if larval activity coincides with high mouse infection prevalence (Fig. 3A. and Fig. 4A.) The temporal concentration of infected hosts is likely to have important consequences for the transmission success and fitness of most pathogens (Altizer et al. 2006; Martinez 2018). 
Extended periods between nymphal and larval activity result in limited transmission efficiency (Fig. 3B and Fig. 4B). This novel prediction for the Lyme disease system is caused by the decay in mouse infection prevalence following nymphal activity due to mouse mortality and the birth of uninfected mice (Hofmeister et al. 1999; Wright et al. 1990). Thus, larvae feeding long after the nymphal activity period have a greater probability of feeding on uninfected mice than those that feed shortly after the nymphal activity period. While high mouse turnover is the norm in this system (Schug et al. 1991), lower mouse turnover would extend the period of high mouse infection prevalence and moderate the declines in parasite fitness caused by extended periods between larval and nymphal emergence.

Parasite fitness is predicted to be greatest when all individuals within each developmental stage feed simultaneously and larvae feed immediately after nymphs. This result relies on the assumption that there is no limit to the number of ticks that can feed on a mouse at any given time. Realistically, the number of ticks per mouse is limited by grooming and foraging behaviors. Incorporating a maximum number of ticks per mouse will alter the prediction that simultaneous emergence within life stages maximizes parasite fitness as most ticks will fail to find an available host, resulting in fewer fed ticks each year and thus a lower $R_{0}$. Further, accounting for spatial aggregation of host-seeking larvae would increase the impact of a maximum number of ticks per mouse (Ostfeld et al. 1996c, a, 2018; Devevey and Brisson 2012). Incorporating this ecological realism will cause intermediate emergence concentrations to result in more infected larvae.

The observed fitness of B. burgdorferi in different Lyme disease foci in North America corresponds qualitatively with model predictions. For example, the relatively continuous activity of both tick developmental stages in the Southeastern USA has been proposed as a factor leading to the relatively low B. burgdorferi fitness observed in the region. In the Midwestern USA, where larvae and nymphs are synchronously active during a limited period, B. burgdorferi transmission is lower than in the Northeastern USA but much greater than where both stages are more continuously active (Fig. 5) (Hamer et al. 2012). The correlation between $B$. burgdorferi fitness observed in nature and the expected fitness differences given the observed phenological patterns suggests that both the duration of seasonal activity and the relative timing of activity periods may impact transmission success and parasite fitness (Figs. 3, 4, 6, 5). However, vector phenology is unlikely the only cause of the differences in B. burgdorferi transmission success among these regions as many other features that are known to impact $B$. burgdorferi also differ including host community composition, tick host preferences, and landscape and climatic features (James and Oliver Jr 1990; LoGiudice et al. 2003; Brisson and Dykhuizen 2004; Ogden et al. 2005b; Brisson et al. 2008; Khatchikian et al. 2012; Vuong et al. 2014; Adalsteinsson et al. 2016; Vuong et al. 2017; Adalsteinsson et al. 2018). Nevertheless, our results add to the body of literature that suggests tick phenology can impact $B$. burgdorferi fitness.

Our model captures the impact of phenology on $B$. burgdorferi transmission and fitness in a much simpler modeling framework than previously published studies that successfully address several hypotheses specific to the system (Ogden et al. 2004, 2008; Dunn et al. 2013). In particular, previous work focused on accurately predicting $B$. burgdorferi incidence given phenological scenarios in several realistic environments that depend upon several dozens of parameters, all of which require empirical validation (Ogden et al. 2004). In contrast, our model has 15 parameters and a straightforward structure. This relative simplicity allows our model to serve as a basis for studying phenological impacts in a broad range of environmental scenarios and disease systems as well as exploring the ramifications of other complicating factors such as the evolutionary dynamics of virulence.

As all disease systems exhibit seasonality, phenological drivers may have large impacts on the transmission success, and disease risk from, many parasites. Geographic variation in host or vector phenology may also be an important driver of documented variations in pathogen prevalence and disease risk (Altizer et al. 2006; Martinez 2018). Public health predictions of disease risk may be improved by accounting for phenological variation. Further, the dramatic shifts in host and vector phenology driven by global climate change (Penuelas 2001; Meyer et al. 2014; Post et al. 2001; Johansson et al. 2014) may result in equally dramatic shifts in pathogen prevalence at regional or global scales.

Author Contributions Hannelore MacDonald and Dustin Brisson conceived of the presented idea; Hannelore MacDonald, Erol Akçay, and Dustin Brisson developed the theoretical framework; Hannelore MacDonald conducted the mathematical analysis and performed numerical simulations; Erol Akçay supervised the mathematical analysis and numerical simulations; All authors wrote the manuscript and gave final approval for publication and agree to be held accountable for the work performed therein.

Funding Information This work was supported by the National Institute of Health (T32AI141393 (HM) R01AI142572 (DB), R01AI097137 (DB)); the National Science Foundation (DEB-1354184 (DB)); and the Burroughs Wellcome Fund (1012376 (DB)).

Data Availability Statement Mathematica code written to generate figures in the main text and appendix are included as Supporting Information. 


\section{Compliance with ethical standards}

Conflicts of interest/Competing interests The authors declare that they have no conflict of interest.

Ethics approval Not applicable

Consent to participate Not applicable

Consent for publication All authors gave final approval for publication.

Open Access This article is licensed under a Creative Commons Attribution 4.0 International License, which permits use, sharing, adaptation, distribution and reproduction in any medium or format, as long as you give appropriate credit to the original author(s) and the source, provide a link to the Creative Commons licence, and indicate if changes were made. The images or other third party material in this article are included in the article's Creative Commons licence, unless indicated otherwise in a credit line to the material. If material is not included in the article's Creative Commons licence and your intended use is not permitted by statutory regulation or exceeds the permitted use, you will need to obtain permission directly from the copyright holder. To view a copy of this licence, visit http://creativecommons.org/licenses/by/4.0/.

\section{Appendix A}

In Appendix A we derive between-season equilibrial solutions for tick demography (A.1a - A.1d), ignoring infection status. The following differential equations describe within-season tick population dynamics, valid from $(0, \tau)$ where $\tau$ is the length of the tick feeding season. For simplicity, we assume that the mouse population is constant so that $M=k\left(1-\frac{\mu_{m}}{b}\right)$ where $b$ is the mouse birth rate, $k$ is the carrying capacity and $\mu_{m}$ is the mouse death rate. All other parameters are the same as described in the main text.

$\frac{d L_{q}}{d t}=\hat{L}(T) g_{l}\left(t, \theta_{l}\right)-L_{q}\left(\gamma_{l} M+\mu_{l}\right)$,

$\frac{d L_{f}}{d t}=\gamma_{l} L_{q} M$,

$\frac{d N_{q}}{d t}=\hat{N}(T) g_{n}\left(t, \theta_{n}\right)-N_{q}\left(\gamma_{n} M+\mu_{n}\right)$,

$\frac{d N_{f}}{d t}=\gamma_{n} N_{q} M$.

(A.1a-d) is solved analytically by describing tick emergence using a uniform distribution $g_{.}\left(t, l_{\bullet}\right)= \begin{cases}0 & t \leq t_{\bullet 0} \\ \frac{1}{l_{0}} & t_{\bullet 0} \leq t \leq t_{\bullet f} \\ 0 & t_{\bullet f} \leq t\end{cases}$

Within-season dynamics are coupled to recurrence equations that describe nymphal and larval survival between years. The total fed larval population at the end of the year, $L_{f}(\tau)$ gives rise to the population of nymphs $\hat{N}$ that will emerge the following year, described by the map

$\hat{N}(T+1)=\sigma_{l} L_{f}(\tau)$

where $\sigma_{l}$ accounts for the survival between fed larvae and questing nymphs and the number of fed larvae at time $\tau$ is given by

$$
\begin{array}{r}
L_{f}(\tau)=\frac{\gamma_{l} \hat{L}(T) M}{l_{l}\left(\gamma_{l} M+\mu_{l}\right)}\left(\int_{t_{l 0}}^{t_{l f}} 1-e^{-\left(\gamma_{l} M+\mu_{l}\right) x} d x\right. \\
+\left(1-e^{-\left(\gamma_{l} M+\mu_{l}\right) l_{l}}\right) \\
\left.\int_{0}^{\tau-t_{l f}} e^{-\left(\gamma_{l} M+\mu_{l}\right) x} d x\right)
\end{array}
$$

Similarly, the total fed nymphal population at the end of the year, $N_{f}(\tau)$, gives rise to the population of larvae $\hat{L}$ that will emerge the following year, described by the map

$\hat{L}(T+1)=\frac{\sigma_{n} N_{f}(\tau)}{\left.1+\alpha N_{f}(\tau)\right)}$

This expression accounts for the expected number of questing larvae produced per nymph that feeds to repletion after accounting for survival through adulthood, density-dependent adult fecundity, and survival from egg to questing larva. The number of fed nymphs at time $\tau$ is given by

$$
\begin{aligned}
N_{f}(\tau) & =\frac{\gamma_{n} \hat{N}(T) M}{l_{n}\left(\gamma_{n} M+\mu_{n}\right)}\left(\int_{0}^{t_{n f}} 1-e^{-\left(\gamma_{n} M+\mu_{n}\right) x} d x\right. \\
& \left.+\left(1-e^{-\left(\gamma_{n} M+\mu_{n}\right) l_{n}}\right) \int_{0}^{\tau-t_{n f}} e^{-\left(\gamma_{n} M+\mu_{n}\right) x} d x\right)
\end{aligned}
$$

If we define 


$$
\begin{aligned}
\phi_{l} & =\frac{\gamma_{l} M}{l_{l}\left(\gamma_{l} M+\mu_{l}\right)}\left(\int_{t_{l 0}}^{t_{l f}} 1-e^{-\left(\gamma_{l} M+\mu_{l}\right) x} d x\right. \\
& \left.+\left(1-e^{-\left(\gamma_{l} M+\mu_{l}\right) l_{l}}\right) \int_{0}^{\tau-t_{l f}} e^{-\left(\gamma_{l} M+\mu_{l}\right) x} d x\right), \\
\phi_{n} & =\frac{\sigma_{n} \gamma_{n} M}{l_{n}\left(\gamma_{n} M+\mu_{n}\right)}\left(\int_{0}^{t_{n f}} 1-e^{-\left(\gamma_{n} M+\mu_{n}\right) x} d x\right. \\
& \left.+\left(1-e^{-\left(\gamma_{n} M+\mu_{n}\right) l_{n}}\right) \int_{0}^{\tau-t_{n f}} e^{-\left(\gamma_{n} M+\mu_{n}\right) x} d x\right)
\end{aligned}
$$

The maps for $\hat{L}(T+1)$ and $\hat{N}(T+1)$ can be written as

$$
\hat{L}(T+1)=\frac{\sigma_{n} \phi_{n} \hat{N}(T)}{1+\alpha \phi_{n} \hat{N}(T)},
$$

$\hat{N}(T+1)=\sigma_{l} \phi_{l} \hat{L}(T)$

The equilibrium population size $\hat{L}^{*}$ is then

$$
\begin{aligned}
\hat{L}(T+2) & =\frac{\sigma_{n} \phi_{n} \hat{N}(T+1)}{1+\alpha \phi_{n} \hat{N}(T+1)}, \\
\hat{L}(T+2) & \left.=\frac{\sigma_{n} \sigma_{l} \phi_{n} \phi_{l} \hat{L}(T)}{1+\alpha \sigma_{l} \phi_{n} \phi_{l} \hat{L}(T)}\right) \\
\hat{L}^{*} & \left.=\frac{\sigma_{n} \sigma_{l} \phi_{n} \phi_{l} \hat{L}^{*}}{1+\alpha \sigma_{l} \phi_{n} \phi_{l} \hat{L}^{*}}\right) \\
\hat{L}^{*} & =\frac{\sigma_{n} \sigma_{l} \phi_{n} \phi_{l}-1}{\alpha \sigma_{l} \phi_{n} \phi_{l}}
\end{aligned}
$$

Similarly for $\hat{N}^{*}$

$$
\begin{aligned}
\hat{N}(T+2) & =\sigma_{l} \phi_{l} \hat{L}(T+1) \\
\hat{N}(T+2) & =\frac{\sigma_{n} \sigma_{l} \phi_{n} \phi_{l} \hat{N}(T)}{1+\alpha \phi_{n} \hat{N}(T)} \\
\hat{N}^{*} & =\frac{\sigma_{n} \sigma_{l} \phi_{n} \phi_{l} \hat{N}^{*}}{1+\alpha \phi_{n} \hat{N}^{*}} \\
\hat{N}^{*} & =\frac{\sigma_{n} \sigma_{l} \phi_{n} \phi_{l}-1}{\alpha \phi_{n}}
\end{aligned}
$$

The stability of these equilibrium points is found by considering the biennial maps of the tick life cycle

$$
\begin{aligned}
& \left.\frac{d \hat{L}(T+2)}{d \hat{L}(T)}\right|_{\hat{L}(T)=L^{*}}=-\frac{\sigma_{n} \sigma_{l} \phi_{n} \phi_{l}}{\left(1+\alpha \sigma_{l} \phi_{n} \phi_{l} \hat{L}^{*}\right)^{2}} \\
& \left.\frac{d \hat{L}(T+2)}{d \hat{L}(T)}\right|_{\hat{L}(T)=L^{*}}=\frac{1}{\sigma_{n} \sigma_{l} \phi_{n} \phi_{l}}
\end{aligned}
$$

This system is stable for $\sigma_{n} \sigma_{l} \phi_{n} \phi_{l}>1 . \sigma_{n}, \phi_{n}$, and $\phi_{l}$ are always less than 1 . Therefore, the stability of the tick demographic equilibrium depends on maintaining $\sigma_{l} \phi_{n} \phi_{l}<\frac{1}{\sigma_{n}}$.

Figures in the main text assume both tick life stages are at equilibrium by using $L^{*}$ and $N^{*}$ for the sizes of emerging tick cohorts at the beginning of the season. $\left(\hat{L}(T)=L^{*}, \hat{N}_{i}(T)+\hat{N}_{u}(T)=N^{*}\right)$.

\section{Appendix B}

In Appendix B we derive analytical solutions for Equations $(2 \mathrm{a}-2 \mathrm{~g})$ from the main text. Equations $(2 \mathrm{a}-2 \mathrm{~g})$ assume that the host population is at equilibrium, $\left(M=k\left(1-\frac{\mu_{m}}{b}\right)\right)$. We put these equations again in Appendix B:

$\frac{d L_{q}}{d t}=\hat{L}(T) g_{l}\left(t, t_{l 0}, l_{l}\right)-L_{q}\left(\gamma_{l} M+\mu_{l}\right)$,

$\frac{d L_{i f}}{d t}=\beta_{m l} \gamma_{l} L_{q} M_{i}$,

$\frac{d L_{u f}}{d t}=\gamma_{l} L_{q}\left(M-\beta_{m l} M_{i}\right)$,

$\frac{d N_{i q}}{d t}=\hat{N}_{i}(T) g_{n}\left(t, t_{n 0}, l_{n}\right)-N_{i q}\left(\gamma_{n} M+\mu_{n}\right)$,

$\frac{d N_{u q}}{d t} \hat{N}_{u}(T) g_{n}\left(t, t_{n 0}, l_{n}\right)-N_{u q}\left(\gamma_{n} M+\mu_{n}\right)$,

$\frac{d N_{f}}{d t}=\gamma_{n} M\left(N_{i q}+N_{u q}\right)$,

$\frac{d M_{i}}{d t}=\beta_{n m} \gamma_{n} N_{i q}\left(M-M_{i}\right)-\mu_{m} M_{i}$.

The system (B.1a-g) is solved analytically by describing tick emergence using a uniform distribution

$g_{\bullet}\left(t, t_{\bullet 0}, l_{\bullet}\right)= \begin{cases}0 & t \leq t_{\bullet 0} \\ \frac{1}{l_{0}} & t_{\bullet 0} \leq t \leq t_{\bullet f} \\ 0 & t_{\bullet f} \leq t\end{cases}$

where $t_{\cdot 0}$ denotes the start of emergence, $l_{\text {. }}$ denotes the length of emergence and $t_{\bullet f}$ denotes the end of emergence 
$\left(t_{\bullet 0}+l_{\bullet}=t_{\bullet f}\right)$. The season begins with the emergence of the nymphs $\left(t_{n 0}=0\right)$. Larval emergence, $t_{l 0}$ can begin concurrently with nymphal emergence $\left(t_{l 0}=0\right)$ or have a start time that is offset relative to nymphs $\left(t_{l 0}>0\right)$.

To solve for the within-season dynamics we first find the time-dependent solutions for questing and fed nymphs. Emerging nymphs are split by their infection status $\left(\hat{N}_{i}(T)\right.$ and $\left.\hat{N}_{u}(T)\right)$ which was determined by whether they were infected during their bloodmeal as larvae in the previous season.

$$
\begin{aligned}
& N_{i q}(t)=\left\{\begin{array}{l}
\frac{\hat{N}_{i}(T)}{l_{n}\left(\gamma_{n} M+\mu_{n}\right)}\left(1-e^{-\left(\gamma_{n} M+\mu_{n}\right) t}\right) \quad 0 \leq t \leq t_{n f} \\
N_{i q}\left(t_{n f}\right) e^{-\left(\gamma_{n} M+\mu_{n}\right) t} \quad t_{n f} \leq t
\end{array}\right. \\
& N_{u q}(t)=\left\{\begin{array}{l}
\frac{\hat{N}_{u}(T)}{l_{n}\left(\gamma_{n} M+\mu_{n}\right)}\left(1-e^{-\left(\gamma_{n} M+\mu_{n}\right) t}\right) \quad 0 \leq t \leq t_{n f} \\
N_{u q}\left(t_{n f}\right) e^{-\left(\gamma_{n} M+\mu_{n}\right) t} \quad t_{n f} \leq t
\end{array}\right. \\
& N_{f}(t)=\left\{\begin{array}{l}
\frac{\gamma_{n} M\left(\hat{N}_{i}(T)+\hat{N}_{u}(T)\right)}{l_{n}\left(\gamma_{n} M+\mu_{n}\right)} \int_{0}^{t_{n f}}\left(1-e^{-\left(\gamma_{n} M+\mu_{n}\right) s}\right) d s \quad 0 \leq t \leq t_{n f} \\
N_{f}\left(t_{n f}\right) \int_{0}^{t-t_{n f}} e^{-\left(\gamma_{n} M+\mu_{n}\right) s} d s \quad t_{n f} \leq t
\end{array}\right.
\end{aligned}
$$

We then use $N_{i q}(t)$ to find the solution for mouse infection dynamics:

$M_{i}(t)= \begin{cases}\frac{\gamma_{n} \beta_{n m} \hat{N}_{i}(T) M}{l_{n}\left(\gamma_{n} M+\mu_{n}\right)} M_{i 1}\left(t, \hat{N}_{i}(T)\right) & 0 \leq t \leq t_{n f} \\ \frac{\gamma_{n} \beta_{n m} \hat{i}_{i}(T) M}{l_{n}\left(\gamma_{n} M+\mu_{n}\right)} M_{i 2}\left(t, \hat{N}_{i}(T)\right) & t_{n f} \leq t\end{cases}$

(B.2d) depends on the activity of questing nymphs and is split by whether nymphs are emerging, $M_{i 1}(t)$ for $0 \leq t \leq t_{n f}$ or have finished emerging, $M_{i 2}(t)$ for $t_{n f}<t<\tau$.

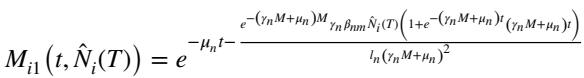

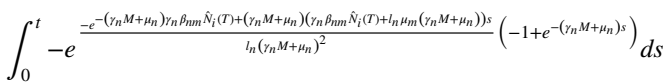

$$
\begin{aligned}
& M_{i 2}\left(t, \hat{N}_{i}(T)\right)=e^{-\mu_{n} t+\frac{e^{-\left(\gamma_{n} M+\mu_{n}\right) t_{n} \beta_{n n} N_{i q}\left(t_{n f}\right)}}{\gamma_{n} M+\mu_{n}}}\left(e^{-\frac{\gamma_{n} \beta_{n n} N_{i q}\left(t_{n f}\right)}{\gamma_{n} M+\mu_{n}}} M_{i 1}\left(t_{n f}, \hat{N}_{i}(T)\right)+\right. \\
& \left.\gamma_{n} \beta_{n m} N_{i q}\left(t_{n f}\right) M \int_{0}^{t} e^{\frac{-e^{-\left(\gamma_{n} M+\mu_{n}\right) s} \gamma_{\gamma_{n}} \beta_{n n} N_{i q}\left(\eta_{n f}\right)}{\gamma_{n} M+\mu_{n}}-\left(\gamma_{n} M-\mu_{m}+\mu_{n}\right) s}\right)
\end{aligned}
$$

We next find the time-dependent solutions for questing and fed larvae, where $\hat{L}(T)$ denotes the emerging larval cohort. We use the solution for $M_{i}(t)$ to split fed larvae by whether they became infected while feeding on a mouse.

$L_{q}(t)=\left\{\begin{array}{l}0 \quad t \leq t_{l 0} \\ \frac{\hat{L}(T)}{l_{l}\left(\gamma_{l} M+\mu_{l}\right)}\left(1-e^{-\left(\gamma_{l} M+\mu_{l}\right) t}\right) \quad t_{l 0} \leq t \leq t_{l f} \\ L_{q}\left(t_{l f}\right) e^{-\left(\gamma_{l} M+\mu_{l}\right) t} \quad t_{l f} \leq t \leq t\end{array}\right.$

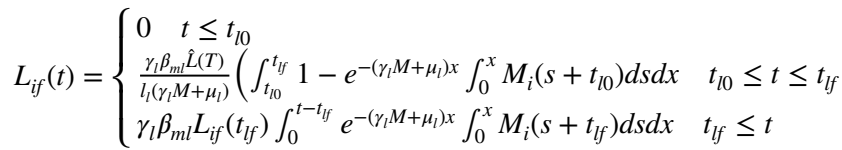

$L_{u f}(t)=\left\{\begin{array}{l}0 \quad t \leq t_{l 0} \\ \frac{\gamma_{l} L(T)}{l_{l}\left(\gamma_{l} M+\mu_{l}\right)}\left(\int_{t_{l 0}}^{t_{l f}} 1-e^{-\left(\gamma_{l} M+\mu_{l}\right) x} \int_{0}^{x}\left(M-\beta_{m l} M_{i}\left(s+t_{l 0}\right)\right) d s d x \quad t_{l 0} \leq t \leq t_{l f}\right. \\ \gamma_{l} L_{u f}\left(t_{l f}\right) \int_{0}^{t-t_{l f}} e^{-\left(\gamma_{l} M+\mu_{l}\right) x} \int_{0}^{x}\left(M-\beta_{m l} M_{i}\left(s+t_{l f}\right)\right) d s d x \quad t_{l f} \leq t\end{array}\right.$

The total number of fed infected larvae by the end of the season, $L_{i f}(\tau)$, fed uninfected larvae by the end of the season, $L_{u f}(\tau)$ and total fed larvae by the end of the season $L_{f}(\tau)$ are given by

$$
\begin{aligned}
L_{i f}(\tau) & =\frac{\gamma_{l} \beta_{m l} \hat{L}(T)}{l_{l}\left(\gamma_{l} M+\mu_{l}\right)}\left(\int_{t_{l 0}}^{t_{l f}} 1-e^{-\left(\gamma_{l} M+\mu_{l}\right) x}\right. \\
& \int_{0}^{x} M_{i}\left(s+t_{l 0}\right) d s d x \\
& +\left(1-e^{-\left(\gamma_{l} M+\mu_{l}\right) l_{l}}\right) \int_{0}^{\tau-t_{l f}} e^{-\left(\gamma_{l} M+\mu_{l}\right) x} \\
& \left.\int_{0}^{x} M_{i}\left(s+t_{l f}\right) d s d x\right) \\
L_{u f}(\tau) & =\frac{\gamma_{l} \hat{L}(T)}{l_{l}\left(\gamma_{l} M+\mu_{l}\right)}\left(\int_{t_{l 0}}^{t_{l f}} 1-e^{-\left(\gamma_{l} M+\mu_{l}\right) x} \int_{0}^{x}\right. \\
& 1-\beta_{m l} M_{i}\left(s+t_{l 0}\right) d s d x \\
& +\left(1-e^{-\left(\gamma_{l} M+\mu_{l}\right) l_{l}}\right) \int_{0}^{\tau-t_{l f}} e^{-\left(\gamma_{l} M+\mu_{l}\right) x} \\
& \left.\int_{0}^{x} 1-\beta_{m l} M_{i}\left(s+t_{l f}\right) d s d x\right) \\
L_{f}(\tau) & =\frac{\gamma_{l} \hat{L}(T) M}{l_{l}\left(\gamma_{l} M+\mu_{l}\right)}\left(\int_{t_{l 0}}^{t_{l f}} 1-e^{-\left(\gamma_{l} M+\mu_{l}\right) x} d x\right. \\
& \left.+\left(1-e^{-\left(\gamma_{l} M+\mu_{l}\right) l_{l}}\right) \int_{0}^{\tau-t_{l f}} e^{-\left(\gamma_{l} M+\mu_{l}\right) x} d x\right)
\end{aligned}
$$

Note that both $L_{i f}(\tau)$ and $L_{u f}(\tau)$ are dependent on $\hat{N}_{i}(T)$ through the transmission dynamics of $M_{i}(t)$.

The total number of fed nymphs by the end of the season is given by 


$$
\begin{aligned}
N_{f}(\tau) & =\frac{\sigma_{n} \gamma_{n}\left(\hat{N}_{i}(T)+\hat{N}_{u}(T)\right) M}{l_{n}\left(\gamma_{n} M+\mu_{n}\right)}\left(\int_{0}^{t_{n f}} 1-e^{-\left(\gamma_{n} M+\mu_{n}\right) x} d x\right. \\
& \left.+\left(1-e^{-\left(\gamma_{n} M+\mu_{n}\right) l_{n}}\right) \int_{0}^{\tau-t_{n f}} e^{-\left(\gamma_{n} M+\mu_{n}\right) x} d x\right)
\end{aligned}
$$

We can also write $L_{i f}(\tau), L_{u f}(\tau)$ and $N_{f}(\tau)$ in terms of the total number of emerging ticks for a given season, $\hat{L}(T)$, $\hat{N}_{i}(T)$, and $\hat{N}_{u}(T)$.

$$
\begin{aligned}
L_{i f}(\tau) & =\phi_{l i}\left(\hat{N}_{i}(T)\right) \hat{L}(T), \\
L_{u f}(\tau) & =\phi_{l u}\left(\hat{N}_{u}(T)\right) \hat{L}(T), \\
N_{f}(\tau) & =\phi_{n} \hat{N}(T) .
\end{aligned}
$$

where $\phi_{n}$ denotes the fraction of emerging nymphs that feed over a season as calculated from within-season dynamics and $\phi_{l i}\left(\hat{N}_{i}(T)\right)$ and $\phi_{l u}\left(\hat{N}_{i}(T)\right)$ are functions of $\hat{N}_{i}(T)$ that denote the fraction of emerging larvae that feed and become infected or remain uninfected, respectively, as calculated from within-season dynamics.

$$
\begin{gathered}
\phi_{l i}\left(\hat{N}_{i}(T)\right)=\frac{\gamma_{l} \beta_{m l}}{l_{l}\left(\gamma_{l} M+\mu_{l}\right)}\left(\int_{t_{l 0}}^{t_{l f}} 1-e^{-\left(\gamma_{l} M+\mu_{l}\right) x}\right. \\
\int_{0}^{x} M_{i}\left(s+t_{l 0}, \hat{N}_{i}(T)\right) d s d x+ \\
\left(1-e^{-\left(\gamma_{l} M+\mu_{l}\right) l_{l}}\right) \int_{0}^{\tau-t_{l f}} e^{-\left(\gamma_{l} M+\mu_{l}\right) x} \\
\left.\int_{0}^{x} M_{i}\left(s+t_{l f}, \hat{N}_{i}(T)\right) d s d x\right), \\
\phi_{l u}\left(\hat{N}_{i}(T)\right)=\frac{\gamma_{l}}{l_{l}\left(\gamma_{l} M+\mu_{l}\right)}\left(\int_{t_{l 0}}^{t_{l f}} 1-e^{-\left(\gamma_{l} M+\mu_{l}\right) x}\right. \\
\int_{0}^{x} 1-\beta_{m l} M_{i}\left(s+t_{l 0}, \hat{N}_{i}(T)\right) d s d x+ \\
\left(1-e^{\left.-\left(\gamma_{l} M+\mu_{l}\right) l_{l}\right)} \int_{0}^{\tau-t_{l f}} e^{-\left(\gamma_{l} M+\mu_{l}\right) x}\right. \\
\left.\int_{0}^{x} 1-\beta_{m l} M_{i}\left(s+t_{l f}, \hat{N}_{i}(T)\right) d s d x\right), \\
\phi_{n}=\frac{\sigma_{n} \gamma_{n} M}{l_{n}\left(\gamma_{n} M+\mu_{n}\right)}\left(\int_{0}^{t_{n f}} 1-e^{-\left(\gamma_{n} M+\mu_{n}\right) x} d x\right. \\
\left.+\left(1-e^{-\left(\gamma_{n} M+\mu_{n}\right) l_{n}}\right) \int_{0}^{\tau-t_{n f}} e^{-\left(\gamma_{n} M+\mu_{n}\right) x} d x\right) .
\end{gathered}
$$

Discrete annual maps of each population can then be written as

$$
\begin{gathered}
\hat{L}(T+1)=\frac{\sigma_{n} \phi_{n} \hat{N}(T)}{\left.1+\alpha \phi_{n} \hat{N}(T)\right)}, \\
\hat{N}_{i}(T+1)=\sigma_{l} \phi_{l i}\left(\hat{N}_{i}(T)\right) \hat{L}(T), \\
\hat{N}_{u}(T+1)=\sigma_{l} \phi_{l u}\left(\hat{N}_{i}(T)\right) \hat{L}(T),
\end{gathered}
$$

To check the stability of tick populations, consider the biennial maps

$$
\begin{aligned}
\hat{L}(T+2) & =\frac{\sigma_{n} \sigma_{l} \phi_{n} \phi_{l} \hat{L}(T)}{1+\alpha \sigma_{l} \phi_{n} \phi_{l} \hat{L}(T)}, \\
\hat{N}_{i}(T+2) & =\frac{\sigma_{l} \sigma_{n} \phi_{n} \phi_{l i}\left(\hat{N}_{i}(T)\right) \hat{N}(T)}{1+\alpha \phi_{n} \hat{N}(T)}, \\
\hat{N}_{u}(T+2) & =\frac{\sigma_{l} \sigma_{n} \phi_{n} \phi_{l u}\left(\hat{N}_{i}(T)\right) \hat{N}(T)}{1+\alpha \phi_{n} \hat{N}(T)}
\end{aligned}
$$

Infection status does not impact demographic rates. Therefore, the larval equilibrium size $\hat{L} *$ and total nymphal equilibrium size $\hat{N}^{*}$ in the infection subsystem are identical to the result found above in Appendix A that ignores infection. $\hat{N}_{i}^{*}$ and $\hat{N}_{u}^{*}$ are both stable for the same conditions given in Appendix A because they are upper bounded by $\hat{N}^{*}\left(\hat{N}_{i}^{*}+\hat{N}_{u}^{*}=\hat{N}^{*}\right)$. Assuming the stability conditions in Appendix A are met, the nontrivial solutions for $\hat{N}_{i}^{*}$ and $\hat{N}_{u}^{*}$ are unique because $\phi_{l i}, \phi_{l u}<1$ and the transmission terms are $0<\beta_{n m}, \beta_{m l}<1$.

$$
\begin{array}{r}
\hat{N}_{i}^{*}=\frac{\sigma_{l} \sigma_{n} \phi_{n} \phi_{l i}\left(\hat{N}_{i}^{*}\right) \hat{N}^{*}}{1+\alpha \phi_{n} \hat{N}^{*}}, \\
\hat{N}_{u} *=\frac{\sigma_{l} \sigma_{n} \phi_{n} \phi_{l u}\left(\hat{N}_{i}^{*}\right) \hat{N}^{*}}{1+\alpha \phi_{n} \hat{N}^{*}}
\end{array}
$$

\section{Appendix C}

In Appendix $\mathrm{C}$ we derive $R_{0}$ to study how phenology impacts thresholds for parasite persistence. $R_{0}$ is computed as the number of infected nymphs that emerge in year $T+1$ produced by a single infected nymph that emerged in year $T$ in an otherwise uninfected population. Specifically, we consider the stability of the disease-free equilibrium when a rare infected nymph is introduced into the tick population, solved by setting $\hat{L}^{*}=\hat{L}(T), \hat{N}^{*}=\hat{N}_{u}(T)-1$, and $\hat{N}_{i}(T)=1$ in equations (20) and (21). 
Three distinct cases of phenological patterns are relevant to this system: (1) Emergence of both tick stages overlaps and larvae finish emerging before nymphs finish emerging. (2) Emergence of both tick stages overlaps and nymphs finish emerging before larvae finish emerging. (3) Nymph emergence ends before larvae emergence begins. Each case needs to be analyzed separately to account for the time-dependent differences in the dynamics.

Using Case 1 as an example to sketch our derivation:

$$
\begin{aligned}
\hat{N}_{i}(T+1) & =\sigma_{l} \phi_{l i}\left(\hat{N}_{i}\right) \hat{L}^{*} \\
\hat{N}_{i}(T+1)= & \frac{\sigma_{l} \gamma_{l} \beta_{m l} \hat{L}^{*}}{l_{l}\left(\gamma_{l} M+\mu_{l}\right)} \frac{\gamma_{n} \beta_{n m} \hat{N}_{i}(T) M}{l_{n}\left(\gamma_{n} M+\mu_{n}\right)} \\
& \left(\int_{0}^{t_{l l}-t_{l 0}} 1-e^{-\left(\gamma_{l} M+\mu_{l}\right) x}\right. \\
& \int_{0}^{x} M_{i 1}\left(s+t_{l 0}, \hat{N}_{i}(T)\right) d s d x \\
& +\int_{0}^{t_{n f}-t_{l f}} e^{-\left(\gamma_{l} M+\mu_{l}\right) x} \int_{0}^{x} M_{i 1} \\
& \left(s+t_{l f}, \hat{N}_{i}(T)\right) d s d x \\
& +\int_{0}^{\tau-t_{n f}} e^{-\left(\gamma_{l} M+\mu_{l}\right)\left(x+t_{n f}-t_{l f}\right)} \\
& \left.\int_{0}^{x} M_{i 2}\left(s, \hat{N}_{i}(T)\right) d s d x\right) \\
R_{0} & =\frac{\hat{N}_{i}(T+1)}{\hat{N}_{i}(T)}=\frac{\sigma_{l} \gamma_{l} \beta_{m l} \hat{L}^{*}}{l_{l}\left(\gamma_{l} M+\mu_{l}\right)} \frac{\gamma_{n} \beta_{n m} M}{l_{n}\left(\gamma_{n} M+\mu_{n}\right)} \\
& \left(\int_{0}^{t_{l f}-t_{l 0}} 1-e^{-\left(\gamma_{l} M+\mu_{l}\right) x} \int_{0}^{x} M_{i 1}\right. \\
& \left.\left(s+t_{l 0}, \hat{N}_{i}(T)\right) d s d x\right) \\
& +\left(\int_{0}^{t_{n f}-t_{l f}} e^{-\left(\gamma_{l} M+\mu_{l}\right) x} \int_{0}^{x} M_{i 1}\right. \\
& \left(s+t_{l f}, \hat{N}_{i}(T)\right) d s d x \\
& +\int_{0}^{\tau-t_{n f}} e^{-\left(\gamma_{l} M+\mu_{l}\right)\left(x+t_{n f}-t_{l f}\right)} \\
& \left.M_{i 2}\left(s, \hat{N}_{i}(T)\right) d s d x\right)
\end{aligned}
$$

for $\hat{N}_{i}(T)=1$, parasites persist in phenological scenarios where $\hat{N}_{i}(T+1) \geq 1$. Parasite fitness is maximized when peak larval activity coincides with peak host prevalence.

When $R_{0}>1$, the number of infected nymphs, $\hat{N}_{i}$, reaches a stable T-periodic equilibrium
$\hat{N}_{i}^{*}=\hat{N}^{*}-\hat{N}_{u}^{*}$

$\hat{N}_{i}^{*}$ for a given phenological scenario can be found by solving for the value of $\hat{N}_{i}$ that satisfies $R_{0}=1$. Again, using Case 1 as an example:

$$
\begin{aligned}
1 & =\sigma_{l}\left(L_{f}(\tau)\right) \frac{\gamma_{l} \beta_{m l} \hat{L}^{*}}{l_{l}\left(\gamma_{l} M+\mu_{l}\right)} \frac{\gamma_{n} \beta_{n m} M}{l_{n}\left(\gamma_{n} M+\mu_{n}\right)}\left(\int_{0}^{t_{l f}-t_{l 0}} 1\right. \\
& \left.-e^{-\left(\gamma_{l} M+\mu_{l}\right) x} \int_{0}^{x} M_{i 1}\left(s+t_{l 0}, \hat{N}_{i}^{*}\right) d s d x\right) \\
& +L_{q}\left(t_{l f}\right) \frac{\gamma_{n} \beta_{n m} M}{l_{n}\left(\gamma_{n} M+\mu_{n}\right)}\left(\int_{0}^{t_{n f}-t_{l f}} e^{-\left(\gamma_{l} M+\mu_{l}\right) x}\right. \\
& \int_{0}^{x} M_{i 1}\left(s+t_{l f}, \hat{N}_{i}^{*}\right) d s d x \\
& \left.+\int_{0}^{\tau-t_{n f}} e^{-\left(\gamma_{l} M+\mu_{l}\right)\left(x+t_{n f}-t_{l f}\right)} \int_{0}^{x} M_{i 2}\left(s, \hat{N}_{i}^{*}\right) d s d x\right)
\end{aligned}
$$

If the total tick population is stable (see Appendix A), $\hat{N}_{i}^{*}$ is upper bounded by $\hat{N}^{*}$ and is therefore stable as well.

\section{Appendix D}

In Appendix D we demonstrate that tick phenological patterns can alter tick equilibrium population sizes and impact $R_{0}$. Not accounting for the impact of phenology on between-season tick population demography by assuming constant tick populations each year leads to over- or underestimates of tick demography (Figure 7) and parasite fitness (Figures 8,9). Our model in contrast accounts for the feedback between vector demography and parasite fitness by considering both within-season transmission and vector population dynamics (see Appendix B and $\mathrm{C}$ ) and between-season vector demography (see Appendix $\mathrm{A}$ and $\mathrm{B}$ ). Mouse density impacts $R_{0}$ (Figure 10 ); the error in $R_{0}$ estimate when not accounting for population dynamics is especially high when mouse density is low.

$R_{0}$ is a function of the equilibrium larval population size, $\hat{L}^{*}$, which is in turn determined by tick phenology (see equation (A.3) in Appendix A).

$R_{0}=\frac{\hat{N}_{i}(T+1)}{\hat{N}_{i}(T)}=\sigma_{l} \phi_{l i}\left(\hat{N}_{i}\right) \hat{L}^{*}$

The ecological feedback between tick phenology, tick demography, and $R_{0}$ is ignored if a constant $\hat{L}$ is assumed for all phenological patterns: 
$R_{0 n c}=\sigma_{l} \phi_{l i}\left(\hat{N}_{i}\right) \hat{L}$

where $R_{0 n c}$ refers to the fact that $R_{0}$ is "not corrected."

The relative error between $R_{0}$ and $R_{0 n c}$ is calculated as $\frac{R_{0 n c}-R_{0}}{R_{0}}$.
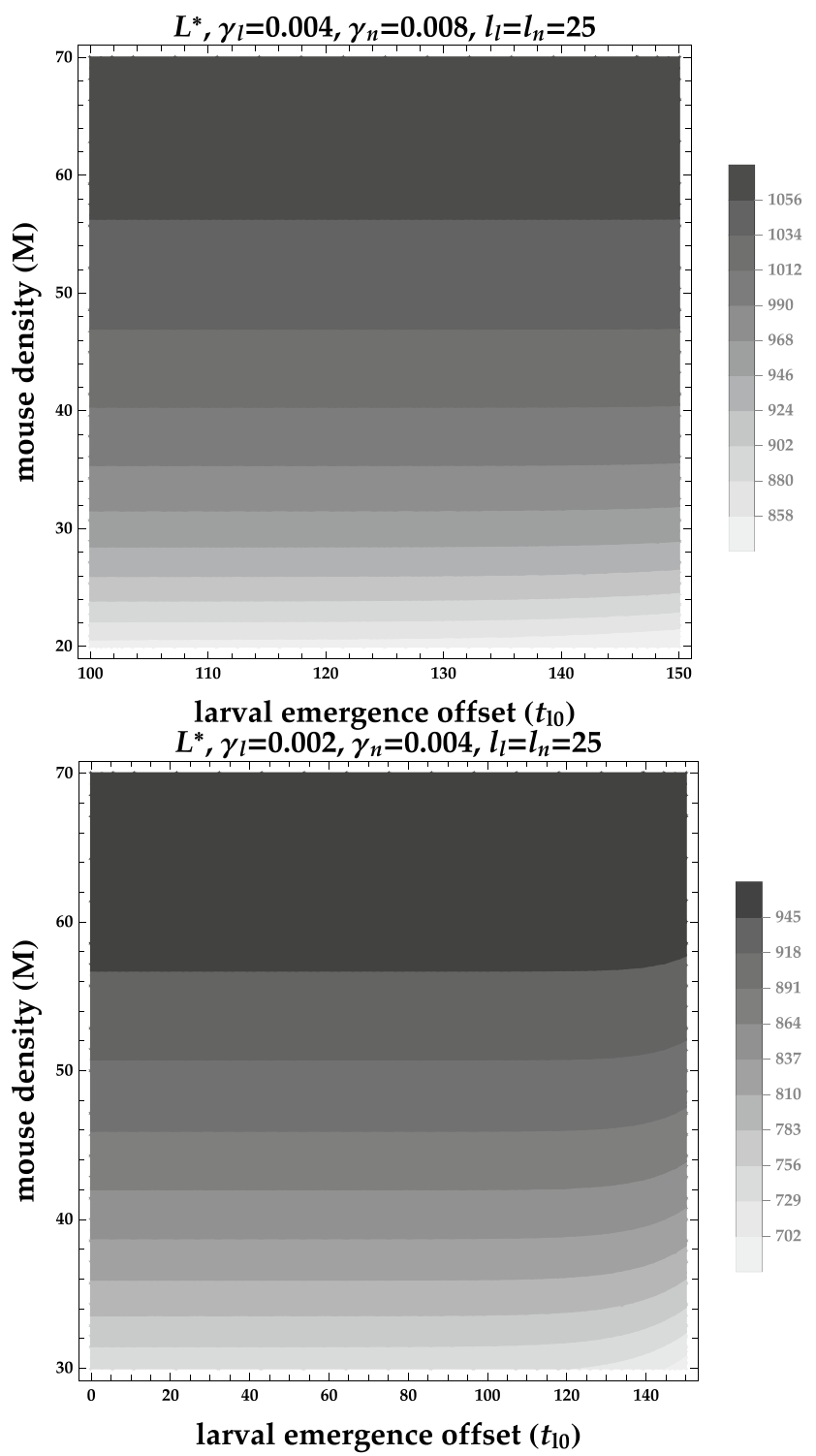

Fig. 7 Equilibrium larval population sizes $\left(\hat{L}^{*}\right)$ decrease at low mouse densities when larval activity begins much later than nymphal activity. $M=k\left(1-\mu_{m} / b\right)$ is the mouse population size, $t_{l 0}$ is the offset between when nymphs and larvae begin emerging, $\gamma_{l}$ and $\gamma_{n}$ are the contact rates between larvae and mice and nymphs and mice, respec-

\section{Appendix E}

In Appendix $\mathrm{E}$ we present numerical simulations of $B$. burgdorferi fitness using the Gamma distribution to describe tick emergence instead of the stricter Uniform distribution. These simulations demonstrate that the shape of the distribution does not significantly change the qualitative results presented in the main text. We also present numerical simulations where we relax the assumption that mouse population sizes are the same across seasons. These
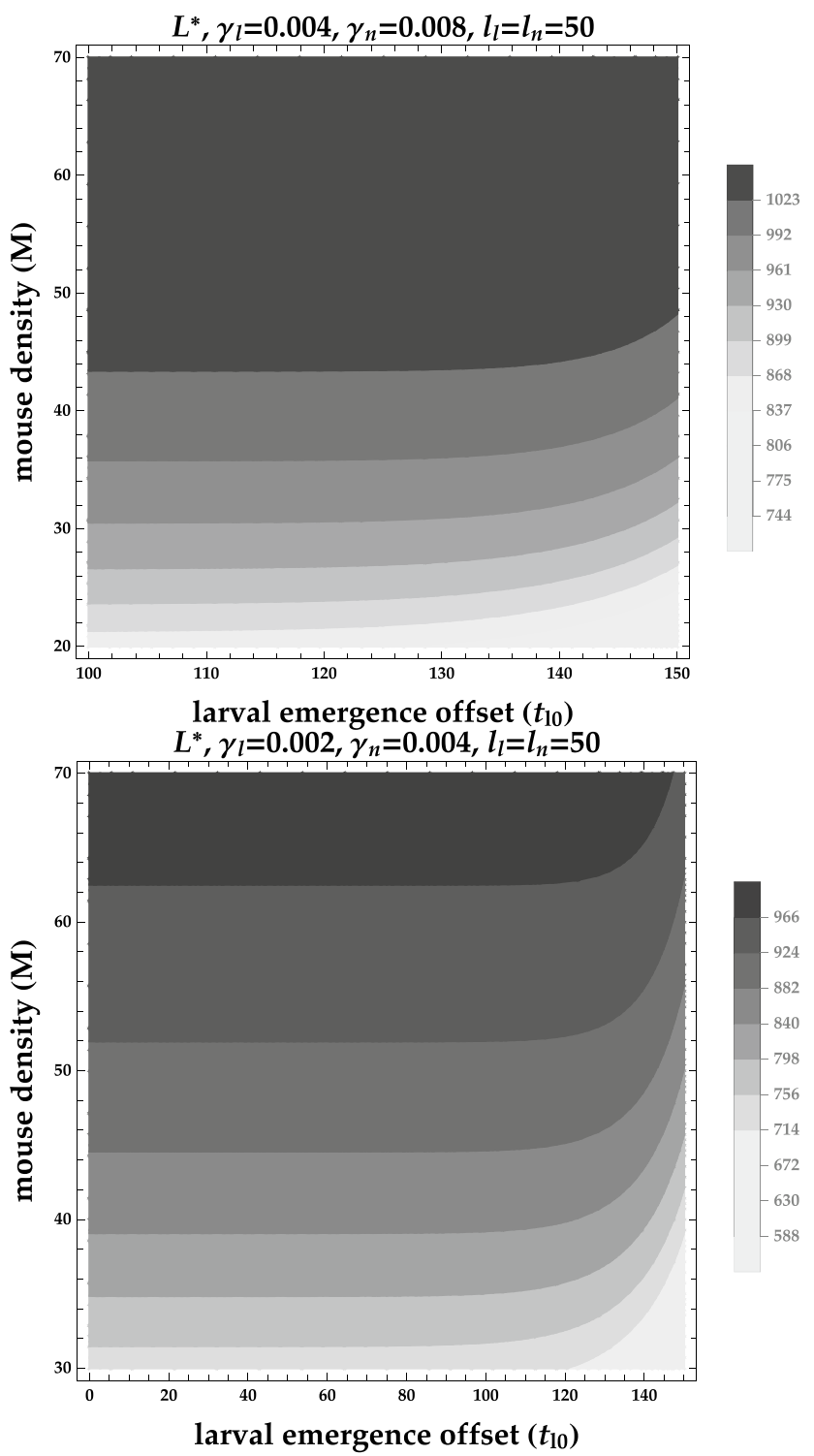

tively. The first row shows that if $\gamma_{l}$ and $\gamma_{n}$ are high, large $t_{l 0}$ decreases $\hat{L}^{*}$ slightly when nymphal and larval emergence is broad $\left(l_{l}\right.$ and $\left.l_{n}\right)$. The second row shows that if $\gamma_{l}$ and $\gamma_{n}$ are low, large $t_{l 0}$ decreases $\hat{L}^{*}$ more strongly, especially when nymphal and larval emergence is broad $\left(l_{l}\right.$ and $\left.l_{n}\right)$. Note that contour colors are not the same across plots. All other parameter values are shown in Table 1 


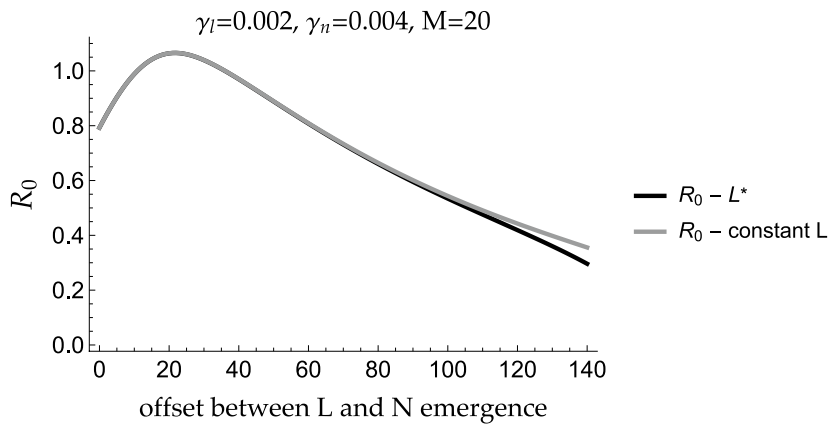

Fig. 8 The difference between $R_{0}$ that takes into account $\hat{L}^{*}$ (black line) and $R_{0 n c}$ that assumes constant $\hat{L}$ regardless of phenology (gray line) is most dramatic for large offset between nymphal and larval activity, low contact rates between ticks and mice $\left(\gamma_{l}=0.002, \gamma_{n}=0.004\right)$ and low mouse density $(M=20)$. The dif-

simulations demonstrate that mouse population size does not alter the results qualitatively.

\section{Gamma distributed tick emergence}

A more natural tick emergence function follows the Gamma distribution:

$g_{\bullet}\left(t, \lambda_{\bullet}, \phi_{\bullet}\right)=\left\{\begin{array}{l}0 \quad t \leq t_{\bullet} \\ \frac{e^{\lambda_{\bullet} t^{\phi_{\bullet}-1}\left(\frac{1}{\lambda_{\bullet}}\right)^{-\phi_{\bullet}}}}{\Gamma\left(\phi_{\bullet}\right)} \quad t_{\bullet 0} \leq t\end{array}\right.$

where emergence begins at $t=t_{\cdot 0}, \phi$. is the shape parameter and $\lambda_{\circ}$ is the scale parameter. We vary $\lambda_{\text {. }}$ in Figures 11 and 12 to alter tick emergence width. Figures 11 and 12 show how $R_{0}$ varies as a function of the time between the start of

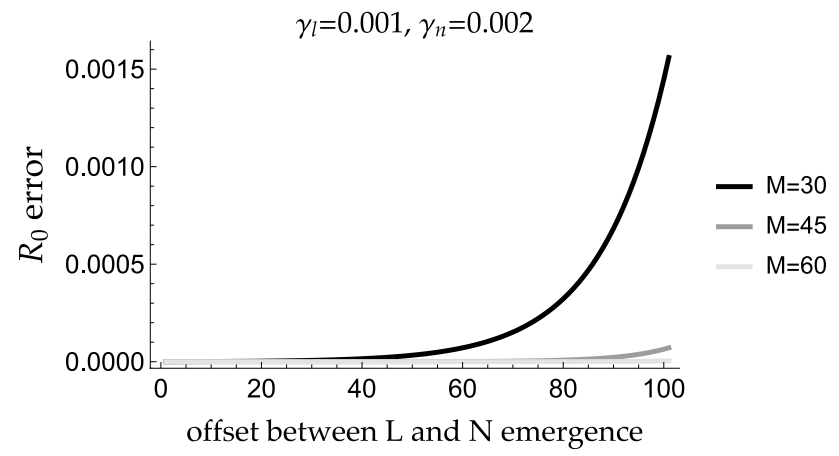

Fig. 9 The relative error between $R_{0}$ and $R_{0 n c}$ increases as the offset between larval and nymphal emergence increases. This error is exacerbated for low host density and low contact rates between ticks and mice because fewer ticks successfully feed by the end of the season which drives lower equilibrium tick sizes. $M=k\left(1-\mu_{m} / b\right)$ is the mouse population size, $\gamma_{l}$ and $\gamma_{n}$ are the contact rates between larvae and mice and nymphs and mice, respectively. All other parameter values are shown in Table 1

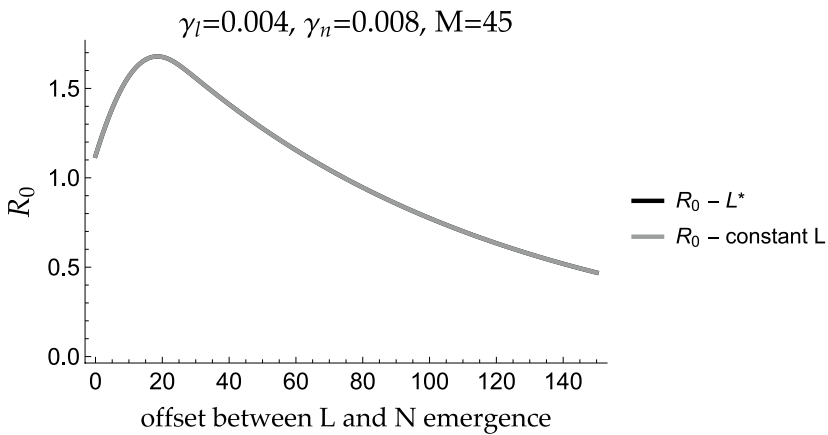

ference between $R_{0}$ and $R_{0 n c}$ is negligible for higher contact rates $\left(\gamma_{l}=0.004, \gamma_{n}=0.008\right)$ and higher mouse density $(M=45) . \hat{L}$ was calculated using the equation (A.3) for $\hat{L}^{*}$ when nymphal and larval phenology is synchronous $\left(t_{l 0}=0\right)$. As the offset between nymphal and larval activity increases, $\hat{L}^{*}$ and $\hat{L}$ diverge driving differences in estimates for $R_{0}$ and $R_{0} n c$

nymphal and larval emergence $\left(t_{l 0}\right)$, the larval emergence width parameter $\left(\lambda_{l}\right)$ and the nymphal emergence width parameter $\left(\lambda_{n}\right)$.

Larval emergence is sometimes bimodal (Brunner and Ostfeld 2008); we thus also model larval emergence using the following distribution:

$g_{l}\left(t, \lambda_{l}, \phi l\right)=\left\{\begin{array}{l}0 \quad t \leq t_{l 0} \\ 1 / 5 \frac{e^{\lambda_{l}\left(t-t_{0}\right)} t^{\phi_{l}-1}\left(\frac{1}{\lambda_{l}}\right)^{-\phi_{l}}}{\Gamma\left(\phi_{l}\right)}+4 / 5 \frac{e^{\lambda_{l}\left(t-t_{l}-10\right)} t^{\phi_{l}-1}\left(\frac{1}{\lambda_{l}}\right)^{-\phi_{l}}}{\Gamma\left(\phi_{l}\right)} t_{l 0} \leq t\end{array}\right.$

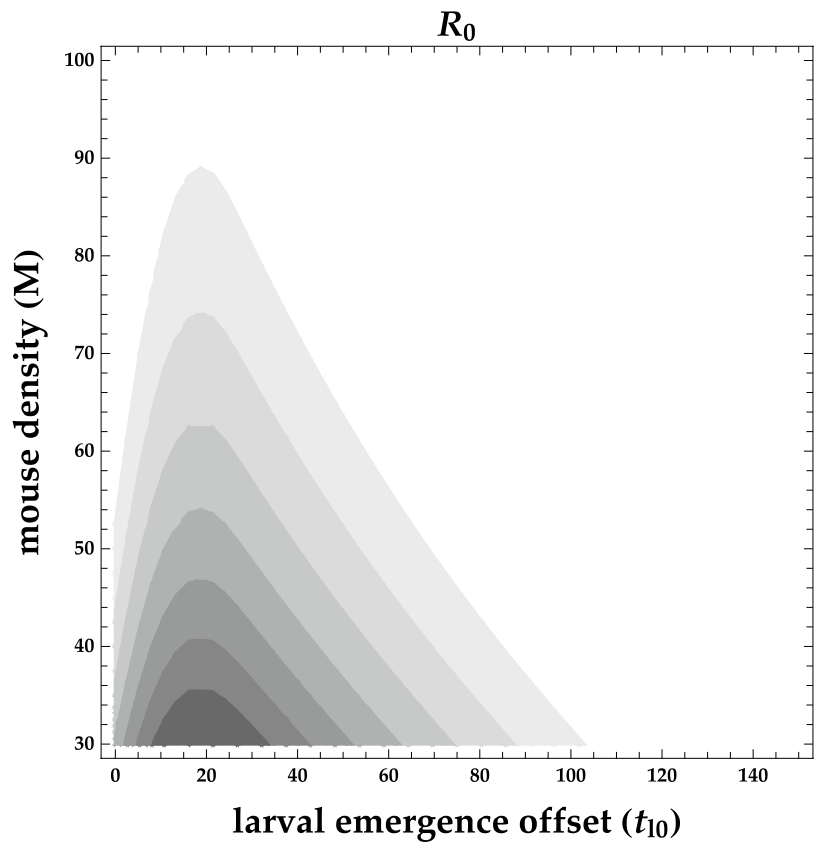

Fig. $10 R_{0}$ decreases as mouse density increases because of dilution. $R_{0}$ decreases when larval activity begins much later than nymphal activity because of mouse turnover 

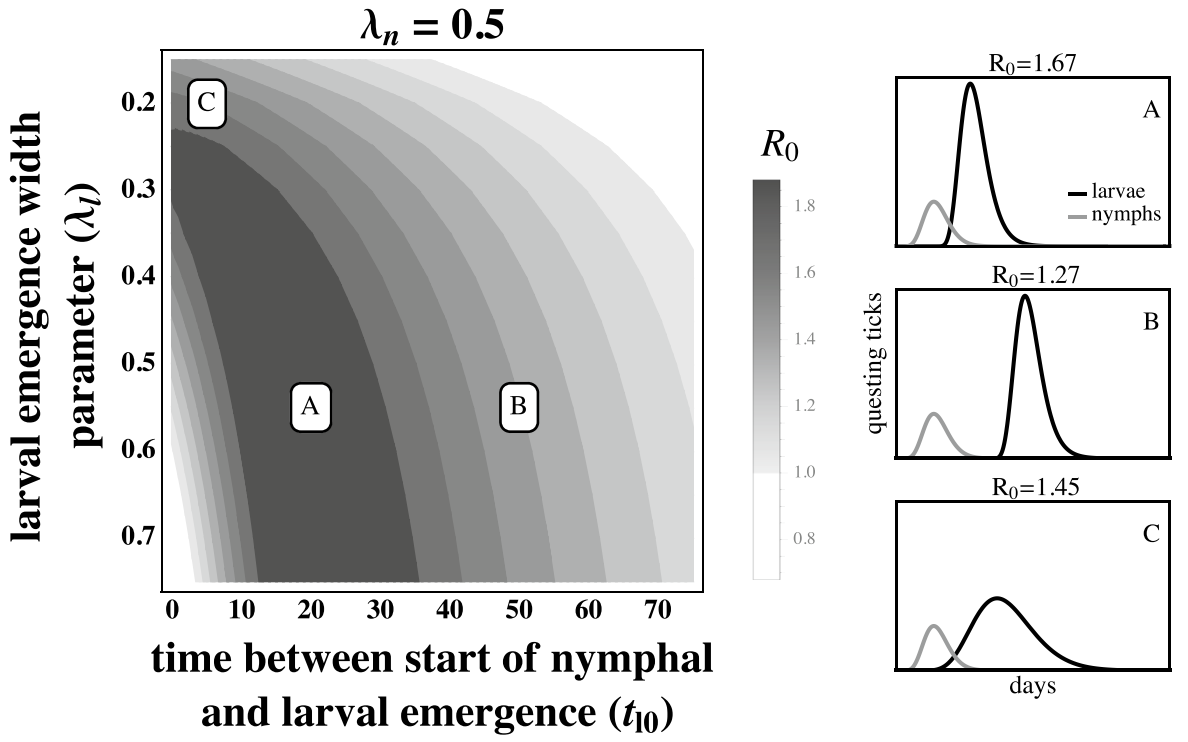

Fig. 11 The basic reproductive number, $R_{0}$, of $B$. burgdorferi is greatest when larval emergence begins shortly after nymphal emergence such that larvae are active during the peak in mouse infection prevalence. The left-hand panel depicts $R_{0}$ as a function of the time between the start of nymphal and larval emergence $\left(t_{l 0}\right)$, and the larval emergence width parameter $\left(\lambda_{l}\right)$, and the letters indicate the parameters for the within-season dynamics on the right-hand panels. (A) Concentrated larval emergence (large $\lambda_{l}$ ) coupled with a slight difference between when nymphs and larvae begin emerging $\left(t_{l 0}<35\right)$ increases the probability that questing larvae feed on mice recently infected by nymphs $\left(t_{l 0}=20, \lambda_{l}=0.55\right) .(B)$ Greater differences between when nymphs and larvae begin emerging $\left(t_{l 0}>35\right)$ result in lower mouse-to-larvae transmission rates as many mice infected by nymphs die and are replaced by mice born uninfected such that larvae are likely to feed on uninfected mice $\left(t_{l 0}=50, \lambda_{l}=0.55\right)$. (C) Synchronous emergence $\left(t_{l 0}=0\right)$ can also reduce $B$. burgdorferi fitness when larval emergence duration is long (small $\lambda_{l}$ ) as many larvae feed after infected mice have died $\left(t_{l 0}=5, \lambda_{l}=0.2\right) . R_{0}$ is calculated assuming tick emergence is Gamma distributed. $\lambda_{n}=0.5, \phi_{l}=\phi_{n}=10, \hat{L}=\hat{L}^{*}, \hat{N}_{i}=1, \hat{N}_{u}=\hat{N}^{*}-1$ (see Appendix A). All other parameter values are shown in Table 1
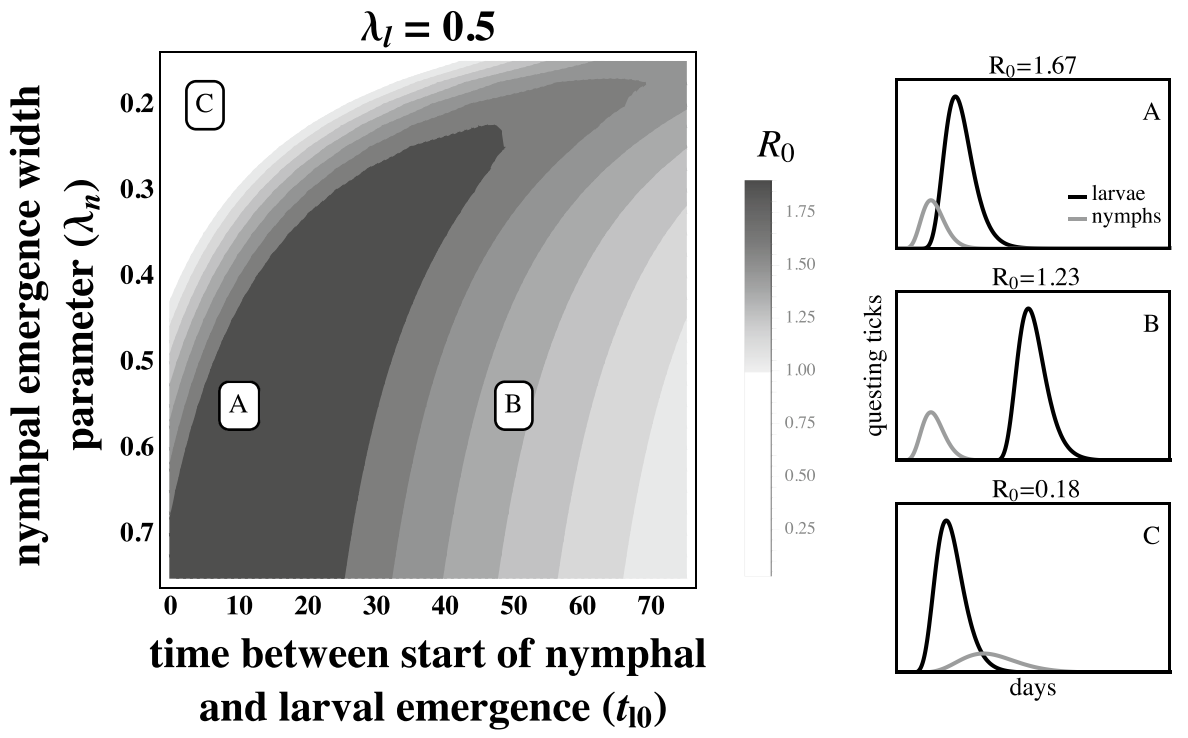

Fig. 12 The basic reproductive number, $R_{0}$, of $B$. burgdorferi is greatest when larval emergence begins shortly after nymphal emergence such that larvae are active during the peak in mouse infection prevalence. The left-hand panel depicts $R_{0}$ as a function of the time between the start of nymphal and larval emergence $\left(t_{10}\right)$, and the nymphal emergence width parameter $\left(\lambda_{n}\right)$, and the letters indicate the parameters for the within-season dynamics on the right-hand panels. (A) Concentrated nymphal emergence (large $\lambda_{n}$ ) coupled with a slight difference between when nymphs and larvae begin emerging $\left(t_{l 0}<25\right)$ increases the probability that questing larvae feed on mice recently infected by nymphs $\left(t_{l 0}=10, \lambda_{n}=0.55\right)$. (B) Greater differences between when nymphs and larvae begin emerging $\left(t_{l 0}>25\right)$ result in lower mouse-to-larvae transmission rates as many mice infected by nymphs die and are replaced by mice born uninfected such that larvae are likely to feed on uninfected mice $\left(t_{l 0}=50, \lambda_{n}=0.55\right)$. (C) Synchronous emergence $\left(t_{l 0}=0\right)$ can also reduce $B$. burgdorferi fitness when nymphal emergence duration is long (small $\lambda_{n}$ ) as many larvae feed before nymphs infect mice $\left(t_{l 0}=5, \lambda_{n}=0.2\right) . R_{0}$ is calculated assuming tick emergence is Gamma distributed. $\lambda_{l}=0.5, \phi_{l}=\phi_{n}=10, \hat{L}=\hat{L}^{*}, \hat{N}_{i}=1, \hat{N}_{u}=\hat{N}^{*}-1$ (see Appendix A). All other parameter values are shown in Table 1 

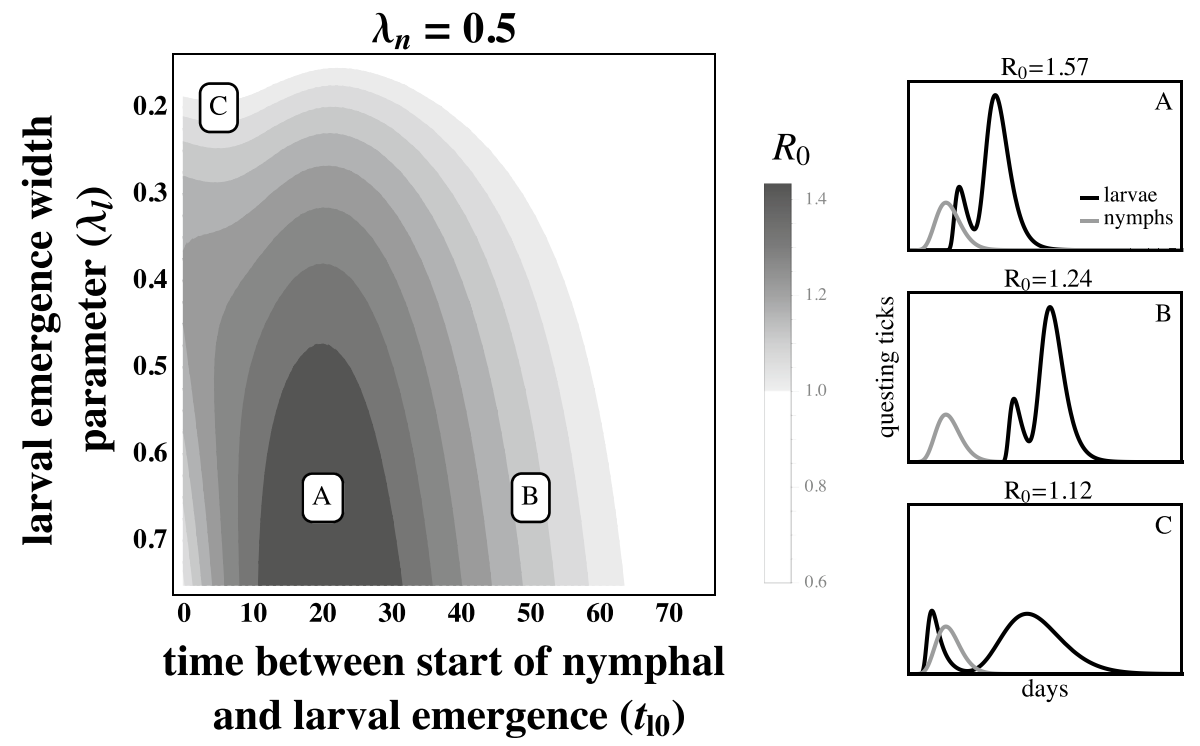

Fig. 13 The basic reproductive number, $R_{0}$, of $B$. burgdorferi is high when larvae emerge shortly after nymphs emerge so that larvae are active when mouse infection prevalence is high, despite bimodal larval emergence. The left-hand panel depicts $R_{0}$ as a function of the time between the start of nymphal and larval emergence $\left(t_{l 0}\right)$, and the larval emergence width parameter $\left(\lambda_{l}\right)$, and the letters indicate the parameters for the within-season dynamics on the right-hand panels. (A) Concentrated larval emergence (large $\lambda_{l}$ ) coupled with a slight difference between when nymphs and larvae begin emerging $\left(t_{l 0}<30\right)$ increases the probability that questing larvae feed on mice recently infected by nymphs $\left(t_{l 0}=20, \lambda_{l}=0.65\right)$. (B) Greater differences between when nymphs and larvae begin emerging $\left(t_{l 0}>30\right)$ result in lower mouse-to-larvae transmission rates as many mice infected by nymphs die and are replaced by mice born uninfected such that larvae are likely to feed on uninfected mice $\left(t_{l 0}=50, \lambda_{l}=0.65\right)$. (C) Synchronous emergence $\left(t_{l 0}=0\right)$ can also reduce $B$. burgdorferi fitness when larval emergence duration is long (small $\lambda_{l}$ ) as many larvae in the first peak feed before nymphs infect mice and many larvae in the later peak feed after infected mice have died $\left(t_{l 0}=5, \lambda_{l}=0.2\right) . R_{0}$ is calculated assuming nymphal emergence is Gamma distributed and larval emergence has a bimodal Gamma distribution. $\lambda_{n}=0.5, \phi_{l}=\phi_{n}=10, \hat{L}=\hat{L}^{*}, \hat{N}_{i}=1, \hat{N}_{u}=\hat{N}^{*}-1$ (see Appendix A). All other parameter values are shown in Table 1
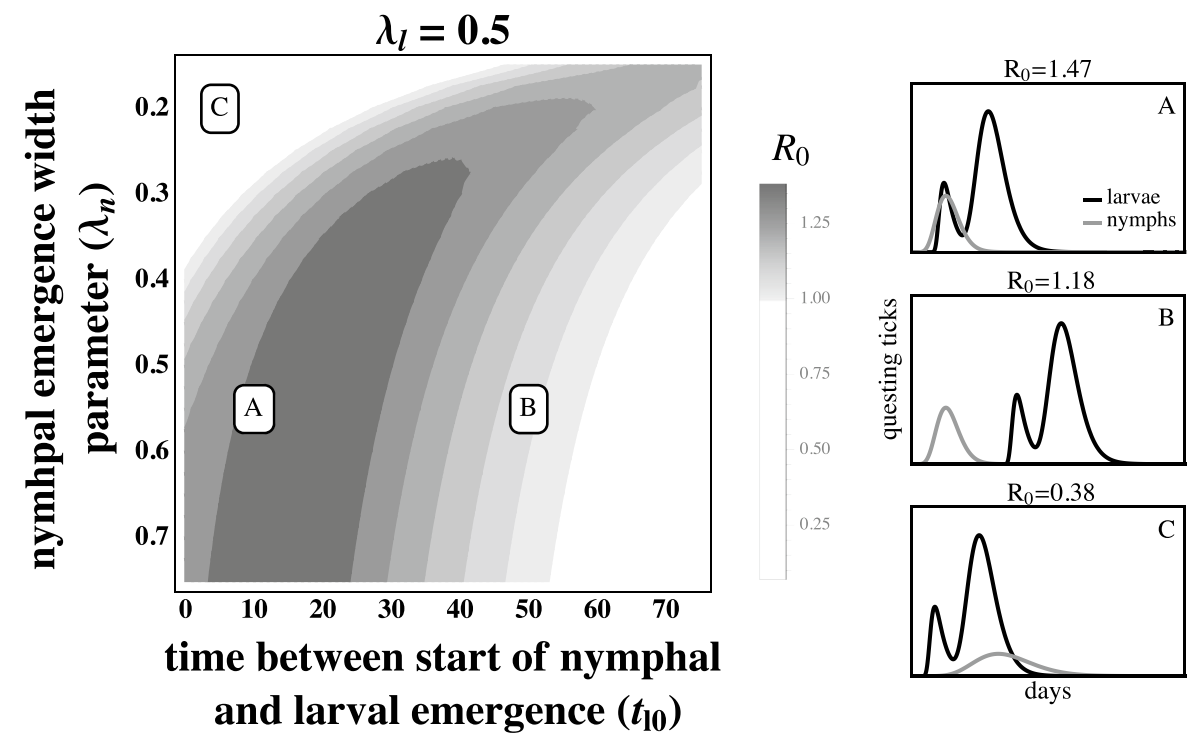

Fig. 14 The basic reproductive number, $R_{0}$, of $B$. burgdorferi is high when larvae emerge shortly after nymphs emerge so that larvae are active when mouse infection prevalence is high, despite bimodal larval emergence. The left-hand panel depicts $R_{0}$, in this case as a function of the time between the start of nymphal and larval emergence $\left(t_{10}\right)$, and the duration of nymphal emergence period $\left(\lambda_{n}\right)$, and the letters indicate the parameters for the within-season dynamics on the right-hand panels. (A) Concentrated nymphal emergence (large $\lambda_{n}$ ) coupled with a slight difference between when nymphs and larvae begin emerging $\left(t_{l 0}<25\right)$ increases the probability that questing larvae feed on mice recently infected by nymphs $\left(t_{l 0}=10, \lambda_{n}=0.55\right)$. (B) Greater dif- ferences between when nymphs and larvae begin emerging $\left(t_{l 0}>25\right)$ result in lower mouse-to-larvae transmission rates as many mice infected by nymphs die and are replaced by mice born uninfected such that larvae are likely to feed on uninfected mice $\left(t_{l 0}=50, \lambda_{n}=0.55\right)$. (C) Synchronous emergence $\left(t_{l 0}=0\right)$ can also reduce $B$. burgdorferi fitness when nymphal emergence duration is long (small $\lambda_{n}$ ) as many larvae feed before nymphs infect mice $\left(t_{l 0}=5, \lambda_{n}=0.2\right.$ ). $R_{0}$ is calculated assuming nymphal emergence is Gamma distributed and larval emergence has a bimodal Gamma distribution. $\lambda_{l}=0.5, \hat{L}=\hat{L}^{*}, \hat{N}_{i}=1, \hat{N}_{u}=\hat{N}^{*}-1$ (see Appendix A). All other parameter values are shown in Table 1 


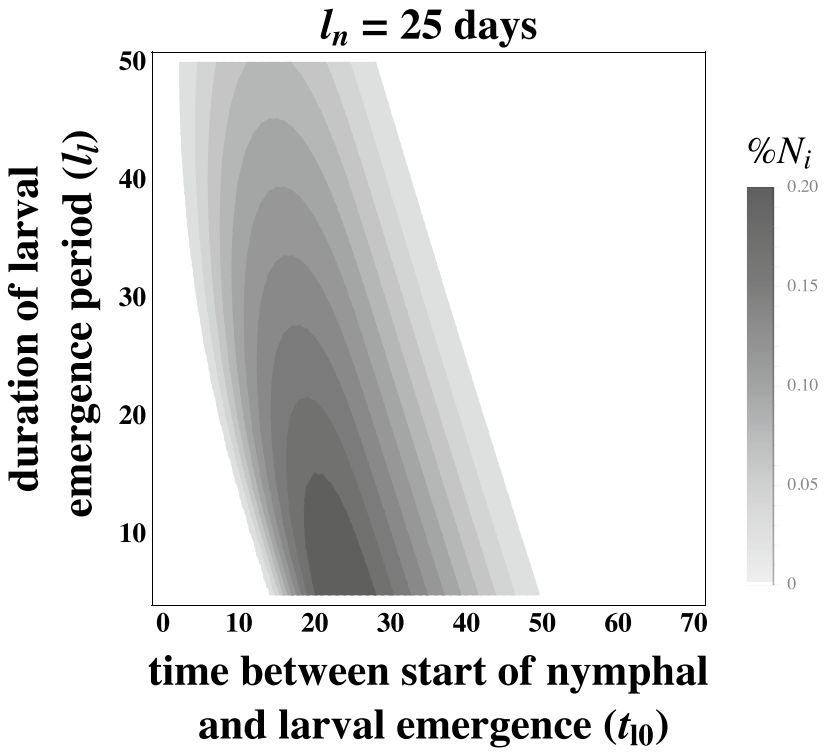

Fig. 15 Nymphal infection prevalence $\left(\% N_{i}\right)$ is highest when larval emergence is concentrated and begins slightly after nymphal emergence despite mouse density varying from one year to the next. This is the same qualitative result as shown in Figures 3 and 4 in the main

Figures 13 and 14 show how $R_{0}$ varies as a function of the time between the start of nymphal and larval emergence $\left(t_{l 0}\right)$, the larval emergence width parameter $\left(\lambda_{l}\right)$ and the nymphal emergence width parameter $\left(\lambda_{n}\right)$ when larval emergence is bimodal.

\section{Variable mouse density}

The main mammalian host of B. burgdorferi, Peromyscus leucopus, often has variable density from year to year, (Ostfeld et al. 1996b). We relax the assumption that mouse density is constant across years by simulating how randomly varying mouse density from one year to the next impacts parasite fitness given different phenological patterns. These results are shown in Figure 15.

\section{References}

Adalsteinsson SA, D’Amico V, Shriver WG, Brisson D, Buler JJ (2016) Scale-dependent effects of nonnative plant invasion on host-seeking tick abundance. Ecosphere 7(3): $\mathrm{e} 01317$

Adalsteinsson SA, Shriver WG, Hojgaard A, Bowman JL, Brisson D, D’Amico V, Buler JJ (2018) Multiflora rose invasion amplifies prevalence of lyme disease pathogen, but not necessarily lyme disease risk. Parasites \& vectors 11(1):54

Altizer S, Dobson A, Hosseini P, Hudson P, Pascual M, Rohani P (2006) Seasonality and the dynamics of infectious diseases. Ecology Letters 9(4):467-484

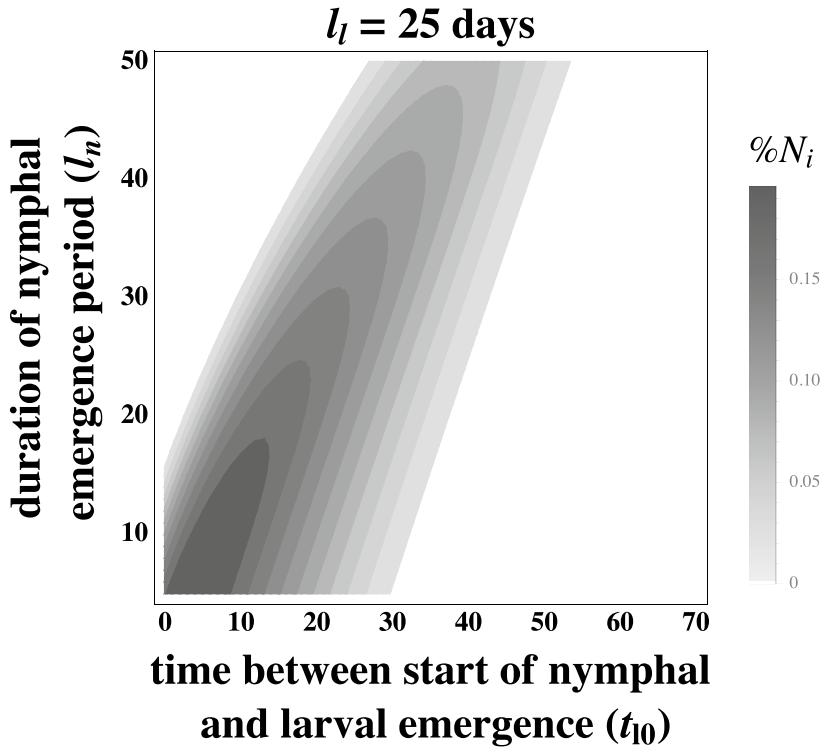

text. $\% N_{i}$ was calculated by taking the geometric mean of nymphal infection prevalence across 180 seasons when mouse density, $M$, randomly varied between 20 and 120 mice. All other parameter values are shown in Table 1

Anderson JF, Johnson RC, Magnarelli LA (1987) Seasonal prevalence of borrelia burgdorferi in natural populations of whitefooted mice, peromyscus leucopus. Journal of clinical microbiology 25(8):1564-1566

Anderson RM (1974) Population dynamics of the cestode caryophyllaeus laticeps (pallas, 1781) in the bream (abramis brama 1.). The Journal of Animal Ecology 305-321

Barber I, Berkhout BW, Ismail Z (2016) Thermal change and the dynamics of multi-host parasite life cycles in aquatic ecosystems. Integrative and comparative biology 56(4):561-572

Bewick S, Cantrell RS, Cosner C, Fagan WF (2016) How Resource Phenology Affects Consumer Population Dynamics. The American Naturalist 187(2):151-166

Brinkerhoff RJ, Folsom-O'Keefe CM, Streby HM, Bent SJ, Tsao K, Diuk-Wasser MA (2011) Regional variation in immature ixodes scapularis parasitism on north american songbirds: implications for transmission of the lyme pathogen, borrelia burgdorferi. Journal of medical entomology 48(2):422-428

Brisson D, Dykhuizen DE (2004) ospc diversity in borrelia burgdorferi: different hosts are different niches. Genetics 168(2):713-722

Brisson D, Dykhuizen DE, Ostfeld RS (2008) Conspicuous impacts of inconspicuous hosts on the lyme disease epidemic. Proceedings of the Royal Society B: Biological Sciences 275(1631):227-235

Brunner JL, Ostfeld RS (2008) Multiple causes of variable tick burdens on small-mammal hosts. Ecology 89(8):2259-2272

Bunikis J, Tsao J, Luke CJ, Luna MG, Fish D, Barbour AG (2004) Borrelia burgdorferi infection in a natural population of peromyscus leucopus mice: a longitudinal study in an area where lyme borreliosis is highly endemic. The Journal of infectious diseases 189(8):1515-1523

Burkett-Cadena ND, McClure CJW, Ligon RA, Graham SP, Guyer C, Hill GE, Ditchkoff SS, Eubanks MD, Hassan HK, Unnasch TR (2011) Host reproductive phenology drives seasonal patterns of host use in mosquitoes. PLoS One 6(3):e17681 
Cahill AE, Aiello-Lammens ME, Fisher-Reid MC, Hua X, Karanewsky CJ, Hae YR, Sbeglia GC, Spagnolo F, Waldron JB, Warsi O et al (2013) How does climate change cause extinction? Proceedings of the Royal Society B: Biological Sciences 280(1750):20121890

Clarke AS (1954) Studies on the life cycle of the pseudophyllidean cestode Schistocephalus solidus. Proceedings of the Zoological Society of London 124(2):257-302

Cleland EE, Chuine I, Menzel A, Mooney HA, Schwartz MD (2007) Shifting plant phenology in response to global change. Trends in Ecology \& Evolution 22(7):357-365

Davis S, Bent SJ (2011) Loop analysis for pathogens: niche partitioning in the transmission graph for pathogens of the north american tick ixodes scapularis. Journal of theoretical biology 269(1):96-103

Devevey G, Brisson D (2012) The effect of spatial heterogenity on the aggregation of ticks on white-footed mice. Parasitology 139(7):915-925

Diuk-Wasser MA, Gatewood AG, Cortinas MR, Yaremych-Hamer S, Tsao J, Kitron U, Hickling G, Brownstein JS, Walker E, Piesman J et al (2006) Spatiotemporal patterns of host-seeking ixodes scapularis nymphs (acari: Ixodidae) in the united states. Journal of medical entomology 43(2):166-176

Dunn JM, Davis S, Stacey A, Diuk-Wasser MA (2013) A simple model for the establishment of tick-borne pathogens of Ixodes scapularis A global sensitivity analysis of R0. Journal of Theoretical Biology 335(C):213-221

Gage KL, Ostfeld RS, Olson JG (1995) Nonviral vector-borne zoonoses associated with mammals in the united states. Journal of Mammalogy 76(3):695-715

Gaku K, Nishikawa Y, Kasagi T, Kosuge S (2004) Does seed production of spring ephemerals decrease when spring comes early? Ecological research 19(2):255-259

Gremillion-Smith C, Woolf A (1988) Epizootiology of skunk rabies in north america. Journal of wildlife diseases 24(4):620-626

Hamer SA, Hickling GJ, Sidge JL, Walker ED, Tsao JI (2012) Synchronous phenology of juvenile ixodes scapularis, vertebrate host relationships, and associated patterns of borrelia burgdorferi ribotypes in the midwestern united states. Ticks and tick-borne diseases 3(2):65-74

Hegland SJ, Nielsen A, Lázaro A, Bjerknes AL, Totland O How does climate warming affect plant-pollinator interactions? Ecology letters 12(2):184-195

Heins DC, Eidam DM, Baker JA (2016) Timing of Infections in the Threespine Stickleback (Gasterosteus aculeatus) by Schistocephalus solidus in Alaska. The Journal of parasitology 102(2):286-289

Hofmeister EK, Ellis BA, Glass GE, Childs JE (1999) Longitudinal study of infection with borrelia burgdorferi in a population of peromyscus leucopus at a lyme disease-enzootic site in maryland. The American journal of tropical medicine and hygiene 60(4):598-609

Hoshen MB, Morse AP (2004) A weather-driven model of malaria transmission. Malaria journal 3(1):32

Inouye DW (2008) Effects of climate change on phenology, frost damage, and floral abundance of montane wildflowers. Ecology 89(2):353-362

James AM, Oliver JH Jr. (1990) Feeding and host preference of immature ixodes dammini, i. scapularis, and i. pacificus (acari: Ixodidae). Journal of Medical Entomology 27(3):324-330

Jepsen JU, Hagen SB, Ims RA, Yoccoz NG (2008) Climate change and outbreaks of the geometrids operophtera brumata and epirrita autumnata in subarctic birch forest: evidence of a recent outbreak range expansion. Journal of Animal Ecology 77(2):257-264

Jepsen JU, Hagen SB, Karlsen SR, Ims RA (2009) Phase-dependent outbreak dynamics of geometrid moth linked to host plant phenology. Proceedings of the Royal Society B: Biological Sciences 276(1676):4119-4128
Johansson J, Nilsson JAA, Jonzén N (2014) Phenological change and ecological interactions: an introduction. Oikos 124(1):1-3

Johnson DM, Büntgen U, Frank DC, Kausrud K, Haynes KJ, Liebhold AM, Esper J, Stenseth NC (2010) Climatic warming disrupts recurrent alpine insect outbreaks. Proceedings of the National Academy of Sciences 107(47):20576-20581

Khatchikian CE, Prusinski M, Stone M, Backenson PB, Wang IN, Levy MZ, Brisson D (2012) Geographical and environmental factors driving the increase in the lyme disease vector ixodes scapularis. Ecosphere 3(10):1-18

Kudo G, Ida TY (2013) Early onset of spring increases the phenological mismatch between plants and pollinators. Ecology 94(10):2311-2320

LoGiudice K, Ostfeld RS, Schmidt KA, Keesing F (2003) The ecology of infectious disease: effects of host diversity and community composition on Lyme disease risk. Proceedings of the National Academy of Sciences 100(2):567-571

Magnarelli LA, Anderson JF, Fish D (1987) Transovarial transmission of Borrelia burgdorferi in Ixodes dammini (Acari:Ixodidae). The Journal of Infectious Diseases 156(1):234-236

Martinez ME (2018) The calendar of epidemics: Seasonal cycles of infectious diseases. PLOS Pathogens 14(11):e1007327-15

McCallum H (2001) How should pathogen transmission be modelled? Trends in Ecology \& Evolution 16(6):295-300

Memmott J, Craze PG, Waser NM, Price MV (2007) Global warming and the disruption of plant-pollinator interactions. Ecology letters 10(8):710-717

Meyer L, Brinkman S, Kesteren Lv, Leprince-Ringuet N, Boxmeer Fv (2014) IPCC, 2014: Climate Change 2014: Synthesis Report. Contribution of Working Groups I, II and III to the Fifth Assessment Report of theIntergovernmental Panel on Climate Change. Technical report Geneva Switzerland

Miller-Rushing AJ, Hoye TT, Inouye DW, Post E (2010) The effects of phenological mismatches on demography. Philosophical Transactions of the Royal Society B: Biological Sciences 365(1555):3177-3186

Ogden NH, Lindsay LR, Beauchamp G, Charron D, Maarouf A, O'Callaghan CJ, Waltner-Toews D, Barker IK (2004) Investigation of relationships between temperature and developmental rates of tick Ixodes scapularis (Acari: Ixodidae) in the laboratory and field. Journal of medical entomology 41(4):622-633

Ogden NH, Bigras-Poulin M, O'Callaghan CJ, Barker IK, Lindsay LR, Maarouf A, Smoyer-Tomic KE, Waltner-Toews D, Charron D (2005a) A dynamic population model to investigate effects of climate on geographic range and seasonality of the tick Ixodes scapularis. International Journal for Parasitology 35(4):375-389

Ogden NH, Bigras-Poulin M, Hanincová K, Maarouf A, O'Callaghan CJ, Kurtenbach K (2008) Projected effects of climate change on tick phenology and fitness of pathogens transmitted by the North American tick Ixodes scapularis. Journal of Theoretical Biology 254(3):621-632

Ogden NH, Bigras-Poulin M, O'Callaghan CJ, Barker IK, Lindsay LR, Maarouf A, Smoyer-Tomic KE, Waltner-Toews D, Charron D (2005b) A dynamic population model to investigate effects of climate on geographic range and seasonality of the tick ixodes scapularis. International journal for parasitology 35(4):375-389

Ogden NH, Pang G, Ginsberg HS, Hickling GJ, Burke RL, Beati L, Tsao JI (2018) Evidence for geographic variation in life-cycle processes affecting phenology of the lyme disease vector ixodes scapularis (acari: Ixodidae) in the united states. Journal of medical entomology 55(6): 1386-1401

Ostfeld RS, Hazler KR, Cepeda OM (1996) Temporal and spatial dynamics of ixodes scapularis (acari: Ixodidae) in a rural landscape. Journal of Medical Entomology 33(1):90-95 
Ostfeld RS, Jones CG, Wolff JO (1996) Of mice and mast. BioScience 46(5):323-330

Ostfeld RS, Miller MC, Hazler KR (1996) Causes and consequences of tick (ixodes scapularis) burdens on white-footed mice (peromyscus leucopus). Journal of Mammalogy 77(1):266-273

Ostfeld RS, Levi T, Keesing F, Oggenfuss K, Canham CD (2018) Tickborne disease risk in a forest food web. Ecology 99(7):1562-1573

Patrican LA (1997) Absence of lyme disease spirochetes in larval progeny of naturally infected ixodes scapularis (acari: Ixodidae) fed on dogs. Journal of Medical Entomology 34(1):52-55

Paull SH, Johnson PTJ (2014) Experimental warming drives a seasonal shift in the timing of host-parasite dynamics with consequences for disease risk. Ecology letters 17(4):445-453

Penuelas J (2001) Phenology: Responses to a Warming World. Science 294(5543):793-795

Piesman J, Donahue JG, Mather TN, Spielman A (1986) Transovarially acquired lyme disease spirochetes (borrelia burgdorferi) in field-collected larval ixodes dammini (acari: Ixodidae). Journal of medical entomology 23(2):219

Post E, Forchhammer MC, Stenseth NC, Callaghan TV (2001) The timing of life-history events in a changing climate. Proceedings of the Royal Society B: Biological Sciences 268(1462):15-23

Powell JA, Bentz BJ (2009) Connecting phenological predictions with population growth rates for mountain pine beetle, an outbreak insect. Landscape Ecology 24(5):657-672

Randolph SE (1999a) Tick ecology: processes and patterns behind the epidemiological risk posed by ixodid ticks as vectors. Parasitology 129(7):S37-S65

Randolph SE (1999b) Tick ecology: processes and patterns behind the epidemiological risk posed by ixodid ticks as vectors. Parasitology 129(7):S37-S65
Randolph SE, Green RM, Peacey MF, Rogers DJ (2000) Seasonal synchrony: the key to tick-borne encephalitis foci identified by satellite data. Parasitology 121(Pt 1):15-23

Schug MD, Vessey SH, Korytko AI (1991) Longevity and Survival in a Population of White-Footed Mice(Peromyscus leucopus). American Society of Mammologists 72(2):360-366

Schwanz LE, Voordouw MJ, Brisson D, Ostfeld RS (2011) Borrelia burgdorferi has minimal impact on the lyme disease reservoir host peromyscus leucopus. Vector-borne and zoonotic diseases 11(2):117-124

van Asch M, Visser ME (2007) Phenology of forest caterpillars and their host trees: the importance of synchrony. Annu Rev Entomol 52:37-55

Vuong HB, Canham CD, Fonseca DM, Brisson D, Morin PJ, Smouse PE, Ostfeld RS (2014) Occurrence and transmission efficiencies of borrelia burgdorferi ospc types in avian and mammalian wildlife. Infection, Genetics and Evolution 27:594-600

Vuong HB, Chiu GS, Smouse PE, Fonseca DM, Brisson D, Morin PJ, Ostfeld RS (2017) Influences of host community characteristics on borrelia burgdorferi infection prevalence in blacklegged ticks. PloS one 12(1):e0167810

Washburn JO, Cornell HV (1981) Parasitoids, patches, and phenology: their possible role in the local extinction of a cynipid gall wasp population. Ecology 62(6):1597-1607

Wilson ML, Spielman A (1985) Seasonal activity of immature ixodes dammini (acari: Ixodidae). Journal of medical entomology 22(4):408-414

Wright SD, Nielson SW (1990) Experimental infection of the whitefooted mouse with borrelia burgdorferi. American journal of veterinary research 51(12):1980-1987 